\title{
ALGEBRAIC VOLUME DENSITY PROPERTY OF AFFINE ALGEBRAIC MANIFOLDS
}

\author{
SHULIM KALIMAN AND FRANK KUTZSCHEBAUCH
}

\begin{abstract}
We introduce the notion of algebraic volume density property for affine algebraic manifolds and prove some important basic facts about it, in particular that it implies the volume density property. The main results of the paper are producing two big classes of examples of Stein manifolds with volume density property. One class consists of certain affine modifications of $\mathbb{C}^{n}$ equipped with a canonical volume form, the other is the class of all Linear Algebraic Groups equipped with the left invariant volume form.
\end{abstract}

\section{INTRODUCTION}

In this paper we study a less developed part of the Andersén-Lempert theory ([1], [3], [7, [20, [18], [19], [6]) namely the case of volume preserving maps. Recall that Andersén-Lempert theory describes complex manifolds such that among other things the local phase flows on their holomorphically convex compact subsets can be approximated by global holomorphic automorphisms which leads to construction of holomorphic automorphisms with prescribed local properties. Needless to say that this implies some remarkable consequences for such manifolds (e.g., see [20], 21], [12]). It turns out that a complex manifold has such approximations if it possesses the following density property introduced by VAROLIN.

1.1. Definition. A complex manifold $X$ has the density property if in the compact-open topology the Lie algebra $\operatorname{Lie}_{\text {hol }}(X)$ generated by completely integrable holomorphic vector fields on $X$ is dense in the Lie algebra $\operatorname{VF}_{\text {hol }}(X)$ of all holomorphic vector fields on $X$. An affine algebraic manifold $X$ has the algebraic density property if the Lie algebra $\operatorname{Lie}_{a l g}(X)$ generated by completely integrable algebraic vector fields on it coincides with the Lie algebra $\mathrm{VF}_{\text {alg }}(X)$ of all algebraic vector fields on it (clearly, the algebraic density property implies the density property).

The algebraic density property was established for a wide variety of affine algebraic manifolds, including all connected linear algebraic groups except for $\mathbb{C}_{+}$and complex

2000 Mathematics Subject Classification. Primary: 32M05,14R20. Secondary: 14R10, 32M25.

Key words and phrases. affine space.

Acknowledgements: This research was started during a visit of the first author to the University of Bern and continued during a visit of the second author to the University of Miami, Coral Gables. We thank these institutions for their generous support and excellent working conditions. The research of the second author was also partially supported by Schweizerische Nationalfonds grant No 200020$124668 / 1$. 
tori by the authors 12. Furthermore, in the coming paper of DonZELLI, Dvorsky and the first author [5] it will be extended to homogeneous affine algebraic manifolds different from $\mathbb{C}_{+}$, complex tori, and one extra surface.

However Andersén, Lempert, Forstneric, Rosay and VArolin considered also another property which has similar consequences for automorphisms preserving a volume form.

1.2. Definition. Let a complex manifold $X$ be equipped with a holomorphic volume form $\omega$ (i.e. $\omega$ is nowhere vanishing section of the canonical bundle). We say that $X$ has the volume density property with respect to $\omega$ if in the compact-open topology the Lie algebra Lie ${ }_{\text {hol }}^{\omega}$ generated by globally integrable holomorphic vector fields $\nu$ such that $\nu(\omega)=0$, is dense in the Lie algebra $\mathrm{VF}_{\text {hol }}^{\omega}(X)$ of all holomorphic vector fields that annihilate $\omega$ (note that condition $\nu(\omega)=0$ is equivalent to the fact that $\nu$ is of zero $\omega$-divergence).

Compared with the density property, the class of complex manifolds with established volume density property has been quite narrow. It was essentially described by the original result of ANDERSÉn and LEMPERT [1, 3] who proved it for Euclidean spaces plus a few other examples found by VAROLIN [20]. In particular he proved that $S L_{2}(\mathbb{C})$ has volume density property with respect to the Haar form but he was unable to decide whether the following hypersurface given by a similar equation like $S L_{2}(\mathbb{C})$

$$
\Sigma^{3}=\left\{(a, b, c, d) \in \mathbb{C}^{4}: a^{2} c-b d=1\right\}
$$

had volume density property or not ([22] section 7$)$.

In order to deal with this lack of examples we introduce like in the previous pattern the following.

1.3. Definition. If $X$ is affine algebraic we say that $X$ has the algebraic volume density property with respect to an algebraic volume form $\omega$ if the Lie algebra $\mathrm{Lie}_{\text {alg }}^{\omega}$ generated by globally integrable algebraic vector fields $\nu$ such that $\nu(\omega)=0$, coincides with the Lie algebra $\mathrm{VF}_{\text {alg }}^{\omega}(X)$ of all algebraic vector fields that annihilate $\omega$.

It is much more difficult to establish the algebraic volume density property than the algebraic density property. This is caused, perhaps, by the following difference which does not allow to apply the most effective criterion for the algebraic density property (see [13]): $\operatorname{VF}_{\text {alg }}^{\omega}(X)$ is not a module over the ring $\mathbb{C}[X]$ of regular functions on $X$ while $\mathrm{VF}_{\text {alg }}(X)$ is. Furthermore, some features that are straightforward for the algebraic density property are not at all clear in the volume-preserving case. For instance, it is not quite obvious that the algebraic volume density property implies the volume density property and that the product of two manifolds with algebraic volume density property has again the algebraic volume density property. We shall show in this paper the validity of these two facts among other results that enable us to enlarge the class of examples of Stein manifolds with the volume density property substantially. In particular we establish the following. 
Theorem 1. Let $X^{\prime}$ be a hypersurface in $\mathbb{C}_{u, v, \bar{x}}^{n+2}$ given by an equation of form $P(u, v, \bar{x})=$ $u v-p(\bar{x})=0$ where $p$ is a polynomial on $\mathbb{C}_{\bar{x}}^{n}$ with a smooth reduced zero fiber $C$ such that its reduced cohomology $\hat{H}^{n-2}(C, \mathbb{C})=0$. Let $\Omega$ be a standard volume form on $\mathbb{C}^{n+2}$ and $\omega^{\prime}$ be a volume form on $X^{\prime}$ such that $\omega^{\prime} \wedge d P=\left.\Omega\right|_{X^{\prime}}$. Then $X^{\prime}$ has the algebraic $\omega^{\prime}$-volume density property.

This gives, of course, an affirmative answer to VAROLIN's question mentioned before. The next theorem is our main result.

Theorem 2. Let $G$ be a linear algebraic group. Then $G$ has the algebraic volume density property with respect to the left (or right) invariant volume form.

Let us describe briefly the content of the paper and the main steps in the proof of these facts.

In Section 2 we remind some standard facts about divergence.

In Section 3 we deal with Theorem 1 in a slightly more general situation. Namely we consider a hypersurface $X^{\prime}$ in $X \times \mathbb{C}_{u, v}^{2}$ given by an equation $P:=u v-p(x)=0$ where $X$ is a smooth affine algebraic variety and $p(x)$ is a regular function on $X$. We suppose that $X$ is equipped with a volume form $\omega$ and establish the existence of a volume form $\omega^{\prime}$ on $X^{\prime}$ such that $\left.\Omega\right|_{X^{\prime}}=d P \wedge \omega$ where $\Omega=d u \wedge d v \wedge \omega$. Then we prove (Proposition 3.3) that $X^{\prime}$ has the $\omega^{\prime}$-volume algebraic density property provided two technical conditions (A1) and (A2) hold.

Condition (A2) is easily verifiable for $X^{\prime}$ and condition (A1) is equivalent to the following (Lemma 3.4): the space of zero $\omega$-divergence algebraic vector fields on $X$ tangent to the zero fiber $C$ of $p$ is generated by vector fields of form $\nu_{1}(f p) \nu_{2}-\nu_{2}(f p) \nu_{1}$ where $\nu_{1}$ and $\nu_{2}$ are commutative completely integrable algebraic vector fields of zero $\omega$-divergence on $X$ and $f$ is a regular function on $X$.

Then we notice the duality between the spaces of zero $\omega$-divergence vector fields on $X$ and closed $(n-1)$-forms on $X$ which is achieved via the inner product that assigns to each vector field $\nu$ the $(n-1)$-form $\iota_{\nu}(\omega)$ (Lemma 3.5). This duality allows to reformulate condition (A1) as the following:

(i) the space of algebraic $(n-2)$-forms on $X$ is generated by the forms of type $\iota_{\nu_{1}} \iota_{\nu_{2}}(\omega)$ where $\nu_{1}$ and $\nu_{2}$ are as before; and

(ii) the outer differentiation sends the space of $(n-2)$-forms on $X$ that vanish on $C$ to the set of $(n-1)$-forms whose restriction to $C$ yield the zero $(n-1)$-form on $C$.

In the case of $X$ isomorphic to a Euclidean space (i) holds automatically with $\nu_{1}$ and $\nu_{2}$ running over the set of partial derivatives.

If the reduced cohomology $\hat{H}^{n-2}(C, \mathbb{C})=0$ and also $H^{n}(X, \mathbb{C})=0$ the validity of (ii) is a consequence of the Grothendieck theorem (see Proposition 3.8) that states that the complex cohomology can be computed via the De Rham complex of algebraic forms on a smooth affine algebraic variety which concludes the proof of Theorem 1.

We end Section 3 with an important corollary of Theorem 1 which will be important for the proof of Theorem 2 : the groups $S L_{2}(\mathbb{C})$ (already proved by VAROLIN as mentioned above) and $P S L_{2}(\mathbb{C})$ have the algebraic volume density property with respect to the invariant volume (Propositions 3.10 and 3.11). The proof is based on the fact that $S L_{2}(\mathbb{C})$ is isomorphic to the hypersurface in $\mathbb{C}_{u, v, x_{1}, x_{2}}^{4}$ given by $u v-x_{1} x_{2}-1=0$. 
Section 4 contains two general facts about the algebraic volume density property with short but non-trivial proofs. The first of them (Proposition 4.1) says that the algebraic volume density property implies the volume density property (in the holomorphic sense). It is also based on the Grothendieck theorem mentioned before. The second one (Proposition 4.4) states that the product $X \times Y$ of two affine algebraic manifolds $X$ and $Y$ with the algebraic volume density property (with respect to volumes $\omega_{X}$ and $\omega_{Y}$ ) has also the algebraic volume density property (with respect to $\omega_{X} \times \omega_{Y}$ ). As a consequence of this result we establish the algebraic volume density property for all tori which was also established earlier by VAROLIN [20] (recall that the density property is not established for higher dimensional tori yet and the algebraic density property does not hold for these objects [2]).

We start Section 5 with discussion of a phenomenon which makes the proof of Proposition 4.4 about the algebraic volume density of $X \times Y$ non-trivial and prevents us from spreading it directly to locally trivial fibrations. More precisely, consider the subspace $F_{Y}$ of $\mathbb{C}[Y]$ generated by the images $\operatorname{Im} \delta$ with $\delta$ running over $\operatorname{Lie}_{\text {alg }}^{\omega}(Y)$. In general $F_{Y} \neq \mathbb{C}[Y]$ and the absence of equality here is the source of difficulties. Nevertherless one can follow the pattern of the proof of Proposition 4.4 in the case of fibrations when the span of $F_{Y}$ and constants yields $\mathbb{C}[Y]$. A manifold $Y$ with this property is called fine and we describe simple facts about such objects which include $S L_{2}$ and $P S L_{2}$. We also introduce the notion of a refined volume fibration $p: W \rightarrow X$ which a generalization of the product situation. Among other assumptions the fiber of $p$, the base $X$, and the total space $W$ are equipped with nicely related volume forms and the fiber is a fine manifold with the algebraic density property. The main result in Section 5 is Theorem 4 saying that the total space of a refined volume fibration has the algebraic volume density property provided its base has the algebraic volume density property as well.

Section 6 contains basic knowledge about invariant volume forms on linear algebraic groups. Of further importance will be Corollary 6.8 about the Mostow decomposition of a linear algebraic group as the product of Levi reductive subgroup and it's unipotent radical. We end it with an important example of a refined volume fibration - the quotient map of a reductive group by its Levi semi-simple subgroup (see Lemma 6.11).

Section 7 prepares the proof of Theorem 2 in the case of a semi-simple group. The central notion discussed in that section is a $p$-compatible vector field $\sigma^{\prime} \in \operatorname{Lie}_{\text {alg }}^{\omega}(W)$ for a locally trivial fibration $p: W \rightarrow X$. Its most important property is that Span Ker $\sigma^{\prime}$. Ker $\delta^{\prime}$ coincides with the algebra $\mathbb{C}[W]$ of regular functions for any $\delta^{\prime} \in \mathrm{VF}_{\text {alg }}^{\omega}(W)$ tangent to the fibers of $p$. It is established that for any at least three-dimensional semi-simple group $G$ and its $S L_{2^{-}}$or $P S L_{2}$-subgroup $S$ corresponding to the root of the Dynkin diagram the fibration $q: G \rightarrow G / S$ admits a sufficiently large family $q$-compatible vector fields. The existence of such a family is an assumption in the definition of a refined fibration which (in combination with the fact that $S L_{2}$ and $P S L_{2}$ are fine and have the algebraic volume density property) leads to the claim that $q$ is refined. This enables us to use properties of refined fibrations established earlier in Proposition 5.16 (but not Theorem 4 since it is unknown whether $G / S$ has the algebraic volume density property). 
Section 8 contains the proof of Theorem 2. The general case follows easily from a semi-simple one (via Lemma 6.11, Theorem 4, and Corollary 6.8). The idea of the proof in the latter case is the following. We consider $S L_{2^{-}}$or $P S L_{2}$-subgroups $S_{0}, \ldots, S_{m}$ corresponding to the simple roots of a Dynkin diagram of a semi-simple group $G$ and fibrations $p_{i}: G \rightarrow G / S_{i}$ with $i=0, \ldots, m$. Using results of Section 7 we establish that there is a sufficiently big collection $\Theta$ of completely integrable fields $\theta$ of zero divergence that are of $p_{i}$-compatible for every $i$. Furthermore up to an element of $\operatorname{Lie}_{\text {alg }}^{\omega}(G)$ every algebraic vector field of zero divergence can be presented as a finite $\operatorname{sum} \sum h_{i} \theta_{i}$ where $\theta_{i} \in \Theta$ and $h_{i} \in \mathbb{C}[G]$. Then we consider a standard averaging operator $\operatorname{av}_{j}$ on $\mathbb{C}[G]$ that assigns to each $h \in \mathbb{C}[G]$ a regular function $\operatorname{av}_{j}(h)$ invariant with respect to the natural $S_{j}$-action on $G$ and establish the following relation: $\sum h_{i} \theta_{i} \in \operatorname{Lie}_{\text {alg }}^{\omega}(G)$ if and only if $\sum \operatorname{av}_{j}\left(h_{i}\right) \theta_{i} \in \operatorname{Lie}_{\text {alg }}^{\omega}(G)$. We show also that a consequent application of operators $\mathrm{av}_{0}, \ldots, \mathrm{av}_{m}$ leads to a function invariant with respect to each $S_{j}, j=0, \ldots, m$. Since the only functions invariant under the natural actions of all such subgroups are constants we see that $\sum h_{i} \theta_{i} \in \operatorname{Lie}_{\text {alg }}^{\omega}(G)$ because $\sum c_{i} \theta_{i} \in \operatorname{Lie}_{\text {alg }}^{\omega}(G)$ for constant coefficients $c_{i}$ which concludes the proof of Theorem 2 ,

The appendix contains definition of strictly semi-compatible fields and refinements of two Lemmas about it from our previous work [12].

Acknowledgments. We would like to thank A. DvOrsky for helpful consultations.

\section{Preliminaries}

Recall that a holomorphic vector field $V \in \mathrm{VF}_{\text {hol }}\left(\mathbb{C}^{n}\right)$ is completely (or globally) integrable if for any initial value $z \in \mathbb{C}^{n}$ there is a global holomorphic solution of the ordinary differential equation

$$
\dot{\gamma}(t)=V(\gamma(t)), \quad \gamma(0)=z .
$$

In this case the phase flow (i.e. the map $\mathbb{C} \times \mathbb{C}^{n} \rightarrow \mathbb{C}^{n}$ given by $\left.(t, z) \mapsto \gamma_{z}(t)\right)$ is a holomorphic action of the additive group $\mathbb{C}_{+}$on $\mathbb{C}^{n}$, where index $z$ in $\gamma_{z}$ denotes the dependence on the initial value. It is worth mentioning that this action is not necessarily algebraic in the case of an algebraic vector field $V \in \mathrm{VF}_{\text {alg }}\left(\mathbb{C}^{n}\right)$.

A holomorphic (resp. algebraic) volume form on a complex (resp. affine algebraic) manifold $X$ of dimension $n$ is a nowhere vanishing holomorphic (resp. algebraic) $n$ -

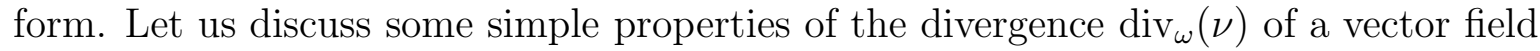
$\nu$ on $X$ with respect to this volume form $\omega$. The divergence is defined by the equation

$$
\operatorname{div}_{\omega}(\nu) \omega=L_{\nu}(\omega)
$$

where $L_{\nu}$ is the Lie derivative. Furthermore, for any vector fields $\nu_{1}, \nu_{2}$ on $X$ we have the following relation between divergence and Lie bracket

$$
\operatorname{div}_{\omega}\left(\left[\nu_{1}, \nu_{2}\right]\right)=L_{\nu_{1}}\left(\operatorname{div}_{\omega}\left(\nu_{2}\right)\right)-L_{\nu_{2}}\left(\operatorname{div}_{\omega}\left(\nu_{1}\right)\right) .
$$

In particular, when $\operatorname{div}_{\omega}\left(\nu_{1}\right)=0$ we have

$$
\operatorname{div}_{\omega}\left(\left[\nu_{1}, \nu_{2}\right]\right)=L_{\nu_{1}}\left(\operatorname{div}_{\omega}\left(\nu_{2}\right)\right)
$$


Another useful formula is

$$
\operatorname{div}_{\omega}(f \nu)=f \operatorname{div}_{\omega}(\nu)+\nu(f)
$$

for any holomorphic function $f$ on $X$.

2.1. Lemma. Let $Y$ be a Stein complex manifold with a volume form $\Omega$ on it, and $X$ be a submanifold of $Y$ which is a strict complete intersection (that is, the defining ideal of $X$ is generated by holomorphic functions $P_{1}, \ldots, P_{k}$ on $Y$, where $k$ is the codimension of $X$ in $Y)$. Suppose that $\nu$ is a vector field on $X$ and $\mu$ is its extension to $Y$ such that $\mu\left(P_{i}\right)=0$ for every $i=1, \ldots, k$. Then

(i) there exists a volume form $\omega$ on $X$ such that $\left.\Omega\right|_{X}=d P_{1} \wedge \ldots \wedge d P_{k} \wedge \omega$; and

(ii) $\operatorname{div}_{\omega}(\nu)=\left.\operatorname{div}_{\Omega}(\mu)\right|_{X}$.

Proof. Let $x_{1}, \ldots, x_{n}$ be a local holomorphic coordinate system in a neighborhood of a point in $X$. Then $P_{1}, \ldots, P_{k}, x_{1}, \ldots, x_{n}$ is a local holomorphic coordinate system in a neighborhood of this point in $Y$. Hence in that neighborhood $\Omega=h d P_{1} \wedge \ldots \wedge d P_{k} \wedge$ $d x_{1} \wedge \ldots \wedge d x_{n}$ where $h$ is a holomorphic function. Set $\omega=\left.h\right|_{X} d x_{1} \wedge \ldots \wedge d x_{n}$. This is the desired volume form in (i).

Recall that $L_{\nu}=d \circ \imath_{\nu}+\imath_{\nu} \circ d$ where $\imath_{\nu}$ is the inner product with respect to $\nu([15]$, Chapter 1, Proposition 3.10). Since $\mu\left(P_{i}\right)=0$ we have $L_{\mu}\left(d P_{i}\right)=0$. Hence by formula (2) we have $\left.\operatorname{div}_{\Omega}(\mu) \Omega\right|_{X}=$

$$
\begin{gathered}
\left.L_{\mu} \Omega\right|_{X}=\left.L_{\mu}\left(d P_{1} \wedge \ldots \wedge d P_{k} \wedge \omega\right)\right|_{X}=\left.d P_{1} \wedge \ldots \wedge d P_{k}\right|_{X} \wedge L_{\nu} \omega+\left.L_{\mu}\left(d P_{1} \wedge \ldots \wedge d P_{k}\right)\right|_{X} \wedge \omega \\
=\left.\operatorname{div}_{\omega}(\nu)\left(d P_{1} \wedge \ldots \wedge d P_{k}\right)\right|_{X} \wedge \omega=\left.\operatorname{div}_{\omega}(\nu) \Omega\right|_{X}
\end{gathered}
$$

which is (ii).

\subsection{Remark.}

(1) Lemma 2.1 remains valid in the algebraic category

(2) Furthermore, it enables us to compute the divergence of a vector field on $X$ via the divergence of a vector field extension on an ambient space. It is worth mentioning that there is another simple way to compute divergence on $X$ which leads to the same formulas in Lemma 2.5 below. Namely, $X$ that we are going to consider will be an affine modification $\sigma: X \rightarrow Z$ of another affine algebraic manifold $Z$ with a volume form $\omega_{0}$ (for definitions of affine and pseudo-affine modifications see [14]). In particular, for some divisors $D \subset Z$ and $E \subset X$ the restriction of $\sigma$ produces an isomorphism $X \backslash E \rightarrow Z \backslash D$. One can suppose that $D$ coincides with the zero locus of a regular (or holomorphic) function $\alpha$ on $Z$. In the situation, we are going to study, the function $\tilde{\alpha}=\alpha \circ \sigma$ has simple zeros on $E$. Consider the form $\sigma^{*} \omega_{0}$ on $X$. It may vanish on $E$ only. Dividing this form by some power $\tilde{\alpha}^{k}$ we get a volume form on $X$. In order to compute divergence of a vector field on $X$ it suffices to find this divergence on the Zariski open subset $X \backslash E \simeq Y \backslash D$, i.e. we need to compute the divergence of a vector 
field $\nu$ on $Y \backslash D$ with respect to a volume form $\beta \omega_{0}$ where $\beta=\alpha^{-k}$. The following formula relates it with the divergence with respect to $\omega_{0}$ :

$$
\operatorname{div}_{\beta \omega_{0}}(\nu)=\operatorname{div}_{\omega_{0}}(\nu)+L_{\nu}(\beta) / \beta .
$$

In the cases, we need to consider, $\beta$ will be often in the kernel of $\nu$, i.e. $\operatorname{div}_{\beta \omega_{0}}(\nu)=$ $\operatorname{div}_{\omega_{0}}(\nu)$ in these cases.

(3) If the normal bundle of $X \subset \mathbb{C}^{n}$ is trivial we may choose $\omega$ as the restriction of the standard volume form on $\mathbb{C}^{n}$ by Lemma 2.1. Indeed, taking $n$ sufficiently large we can always assume that $X$ is a complete intersection in $\mathbb{C}^{n}$ (see for example [17]).

The condition in Lemma 2.1 that an algebraic field $\nu$ on $X$ has an extension $\mu$ on $Y$ with $\mu\left(P_{i}\right)=0$ is also very mild. We consider it in the case of hypersurfaces only.

2.3. Lemma. Let $X$ be a smooth hypersurface in a complex Stein (resp. affine algebraic) manifold $Y$ given by zero of a reduced holomorphic (resp. algebraic) function $P$ on $Y$. Then every holomorphic (resp. algebraic) vector field $\nu$ on $X$ has a similar extension $\mu$ to $Y$ such that $\mu(P)=0$.

Proof. Consider, for instance the algebraic case, i.e. $P$ belongs to the ring $\mathbb{C}[Y]$ of regular functions on $Y$. Since $\mu$ must be tangent to $X$ we see that $\mu(P)$ vanishes on $X$, i.e. $\mu(P)=P Q$ where $Q \in \mathbb{C}[Y]$. Any other algebraic extension of $\nu$ is of form $\tau=\mu-P \theta$ where $\theta \in \mathrm{VF}_{\text {alg }}(Y)$. Thus if $\theta(P)=Q$ then we are done.

In order to show that such $\theta$ can be found consider the set $M=\left\{\theta(P) \mid \theta \in \operatorname{VF}_{\text {alg }}(Y)\right\}$. One can see that $M$ is an ideal of $\mathbb{C}[Y]$. Therefore, it generates a coherent sheaf $\mathcal{F}$ over $Y$. The restriction $\left.Q\right|_{Y \backslash X}$ is a section of $\left.\mathcal{F}\right|_{Y \backslash X}$ because $Q=\mu(P) / P$. Since $X$ is smooth for every point $x \in X$ there are a Zariski open neighborhood $U$ in $Y$ and an algebraic vector field $\partial$ such that $\partial(P)$ does not vanish on $U$. Hence $\left.Q\right|_{U}$ is a section of $\left.\mathcal{F}\right|_{U}$. Since $\mathcal{F}$ is coherent this implies that $Q$ is a global section of $\mathcal{F}$ and, therefore, $Q \in M$ which is the desired conclusion.

2.4. Terminology and Notation. In the rest of this section $X$ is a closed affine algebraic submanifold of $\mathbb{C}^{n}, \omega$ is an algebraic volume form on $X, p$ is a regular function on $X$ such that the divisor $p^{*}(0)$ is smooth reduced, $X^{\prime}$ is the hypersurface in $Y=$ $\mathbb{C}_{u, v}^{2} \times X$ given by the equation $P:=u v-p=0.1$ Note that $X^{\prime}$ is smooth and, therefore, Lemma 2.3 is applicable. We shall often use the fact that every regular function $f$ on $X^{\prime}$ can be presented uniquely as the restriction of a regular function on $Y$ of the form

$$
f=\sum_{i=1}^{m}\left(a_{i} u^{i}+b_{i} v^{i}\right)+a_{0}
$$

\footnotetext{
${ }^{1} \mathrm{By}$ abusing notation we treat $p$ in this formula as a function on $Y$, and, if necessary, we treat it as a function on $X^{\prime}$. Furthermore, by abusing notation, for any regular function on $X$ we denote its lift-up to $Y$ or $X^{\prime}$ by the same symbol.
} 
where $a_{i}=\pi^{*}\left(a_{i}^{0}\right), b_{i}=\pi^{*}\left(b_{i}^{0}\right)$ are lift-ups of regular functions $a_{i}^{0}, b_{i}^{0}$ on $X$ via the natural projection $\pi: Y \rightarrow X$ (as we mentioned by abusing terminology we shall say that $a_{i}$ and $b_{i}$ themselves are regular functions on $X$ ).

Let $\Omega=d u \wedge d v \wedge \omega$, i.e. it is a volume form on $Y$. By Lemma 2.1 there is a volume form $\omega^{\prime}$ on $X^{\prime}$ such that $\left.\Omega\right|_{X^{\prime}}=d P \wedge \omega^{\prime}$. Furthermore, for any vector field $\mu$ such that $\mu(P)=0$ and $\nu^{\prime}=\left.\mu\right|_{X^{\prime}}$ we have $\operatorname{div}_{\omega^{\prime}}\left(\nu^{\prime}\right)=\left.\operatorname{div}_{\Omega}(\mu)\right|_{X}$. Note also that any vector field $\nu$ on $X$ generates a vector field $\kappa$ on $Y$ that annihilates $u$ and $v$. We shall always denote $\left.\kappa\right|_{X^{\prime}}$ by $\tilde{\nu}$. It is useful to note for further computations that $u^{i} \pi^{*}\left(\operatorname{div}_{\omega}(\nu)\right)=\operatorname{div}_{\Omega}\left(u^{i} \kappa\right)$ for every $i \geq 0$. Note also that every algebraic vector field $\lambda$ on $X^{\prime}$ can be written uniquely in the form

$$
\lambda=\tilde{\mu}_{0}+\sum_{i=1}^{m}\left(u^{i} \tilde{\mu}_{i}^{1}+v^{i} \tilde{\mu}_{i}^{2}\right)+f_{0} \partial / \partial u+g_{0} \partial / \partial v
$$

where $\mu_{0}, \mu_{i}^{j}$ are algebraic vector fields on $X$, and $f_{0}, g_{0}$ are regular functions on $X^{\prime}$.

For any algebraic manifold $Z$ with a volume form $\omega$ we denote by $\operatorname{Lie}_{\text {alg }}(Z)$ (resp. $\operatorname{Lie}_{\text {alg }}^{\omega}(Z)$ ) the Lie algebra generated by algebraic globally integrable vector fields on $Z$ (resp. that annihilates $\omega$ ) and by $\mathrm{VF}_{\text {alg }}(Z)$ we denote the Lie algebra of all algebraic vector fields on $Z$. We have a linear map

$$
\widetilde{\operatorname{Pr}}: \mathrm{VF}_{\text {alg }}\left(X^{\prime}\right) \rightarrow \mathrm{VF}_{\text {alg }}(X)
$$

defined by $\widetilde{\operatorname{Pr}}(\lambda)=\mu_{0}$ where $\lambda$ and $\mu_{0}$ are from formula (8). As it was mentioned in [13] the following facts are straightforward calculations that follow easily from Lemma 2.1 ,

2.5. Lemma. Let $\nu_{1}, \nu_{2}$ be vector fields on $X$, and $f$ be a regular function on $X$. For $i \geq 0$ consider the algebraic vector fields

$$
\nu_{1}^{\prime}=u^{i+1} \tilde{\nu}_{1}+u^{i} \nu_{1}(p) \partial / \partial v, \quad \nu_{2}^{\prime}=v^{i+1} \tilde{\nu}_{2}+v^{i} \nu_{2}(p) \partial / \partial u
$$

and $\mu_{f}=f(u \partial / \partial u-v \partial / \partial v)$ on $Y$. Then

(i) $\nu_{i}^{\prime}$ and $\mu_{f}$ are tangent to $X^{\prime}$ (actually they are tangent to fibers of $P=u v-p(x)$ ), i.e., they can be viewed as vector fields on $X^{\prime}$;

(ii) $\mu_{f}$ is always globally integrable on $X^{\prime}$, and $\nu_{i}^{\prime}$ is globally integrable on $X^{\prime}$ if $\nu_{i}$ is globally integrable on $X$;

(iii) $\operatorname{div}_{\omega^{\prime}}\left(\mu_{f}\right)=0, \operatorname{div}_{\omega^{\prime}}\left(\nu_{1}^{\prime}\right)=u^{i+1} \operatorname{div}_{\omega}\left(\nu_{1}\right), \operatorname{div}_{\omega^{\prime}}\left(\nu_{2}^{\prime}\right)=v^{i+1} \operatorname{div}_{\omega}\left(\nu_{2}\right)$, and

$$
\operatorname{div}_{\omega^{\prime}}\left(\left[\mu_{f}, \nu_{1}^{\prime}\right]=(i+1) u^{i+1} f \operatorname{div}_{\omega}\left(\nu_{1}\right), \operatorname{div}_{\omega^{\prime}}\left(\left[\nu_{2}^{\prime}, \mu_{f}\right]=(i+1) v^{i+1} f \operatorname{div}_{\omega}\left(\nu_{2}\right) ;\right.\right.
$$

(iv) we have the following Lie brackets

$$
\begin{aligned}
& {\left[\mu_{f}, \nu_{1}^{\prime}\right]=(i+1) u^{i+1} f \tilde{\nu}_{1}+\alpha_{1} \partial / \partial u+\beta_{1} \partial / \partial v,} \\
& {\left[\nu_{2}^{\prime}, \mu_{f}\right]=(i+1) v^{i+1} f \tilde{\nu}_{2}+\alpha_{2} \partial / \partial u+\beta_{2} \partial / \partial v,}
\end{aligned}
$$

where $\alpha_{i}$ and $\beta_{i}$ are some regular functions on $X^{\prime}$;

(v) more precisely, if $i=0$ in formulas for $\nu_{1}^{\prime}$ and $\nu_{2}^{\prime}$ then

$$
\left[\mu_{f}, \nu_{1}^{\prime}\right]=f u \tilde{\nu}_{1}-u^{2} \nu_{1}(f) \partial / \partial u+\nu_{1}(f p) \partial / \partial v,
$$




$$
\left[\nu_{2}^{\prime}, \mu_{f}\right]=f v \tilde{\nu}_{2}-v^{2} \nu_{2}(f) \partial / \partial v+\nu_{2}(f p) \partial / \partial u
$$

and

$$
\widetilde{\operatorname{Pr}}\left(\left[\left[\mu_{f}, \nu_{1}^{\prime}\right], \nu_{2}^{\prime}\right]\right)=\nu_{1}(f p) \nu_{2}-\nu_{2}(f p) \nu_{1}+f p\left[\nu_{1}, \nu_{2}\right]
$$

\section{The proof of Theorem 1.}

3.1. Additional Notation. For every affine algebraic manifold $Z$ let $\mathbb{C}[Z]$ be the algebra of its regular functions, $\operatorname{IVF}_{\text {alg }}(Z)$ be the set of completely integrable algebraic vector fields on $Z$. If there is a volume form $\omega$ on $Z$ then we denote by $\operatorname{Div}_{Z}$ : $\operatorname{VF}_{\text {alg }}(Z) \rightarrow \mathbb{C}[Z]$ the map that assigns to each vector field its divergence with respect

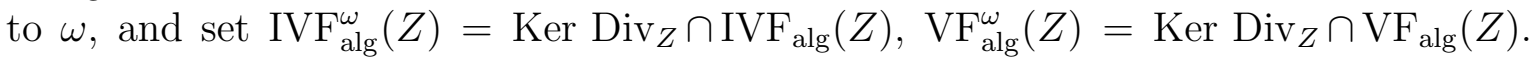
For a closed submanifold $C$ of $Z$ denote by $\operatorname{VF}_{\text {alg }}^{\omega}(Z, C)$ the Lie algebra of vector fields of zero divergence on $Z$ that are tangent to $C$. Formula (7) yields a monomorphism of vector spaces $\iota: \mathbb{C}\left[X^{\prime}\right] \hookrightarrow \mathbb{C}[Y]$ and the natural embedding $X \hookrightarrow X \times(0,0) \subset Y$ generates a projection $\operatorname{Pr}: \mathbb{C}[Y] \rightarrow \mathbb{C}[X]$. Note that $\operatorname{Pr}(\iota(f))=a_{0}$ in the notation of formula (77).

3.2. Lemma. Let $\lambda$ be a vector field on $X^{\prime} \subset X \times \mathbb{C}_{u, v}^{2}$ given by formula (8). Suppose that $\omega_{0}$ is a volume form on $X$ and a volume form $\omega$ on $X^{\prime}$ coincides with the pull-back of the volume form $\omega_{1}:=\left(\omega_{0} \wedge d u\right) / u$ on $Z:=X \times \mathbb{C}_{u}$ under the natural projection (i.e. $\omega$ constructed as in Remark 2.2). Then $\operatorname{Pr}\left(\operatorname{div}_{\omega}(\lambda)\right)=\operatorname{div}_{\omega_{0}}\left(\mu_{0}\right)$. In particular, if $\operatorname{div}_{\omega} \lambda=0$ then $\operatorname{div}_{\omega_{0}}\left(\mu_{0}\right)=0$.

Proof. The natural projection $\sigma: X^{\prime} \rightarrow Z$ is an affine modification whose restriction over $X \times \mathbb{C}_{u}^{*}$ is an isomorphism. Hence $\lambda$ is the pull-back of the following vector field

$$
\kappa=\tilde{\mu}_{0}+\sum_{i=1}^{m} u^{i}\left(\tilde{\mu}_{i}^{1}+\tilde{\mu}_{i}^{2} / p^{i}\right)+f_{0} \partial / \partial u
$$

on $Z$. Thus it suffices to show that $\operatorname{div}_{\omega_{0}}\left(\mu_{0}\right)=T_{0}\left(\operatorname{div}_{\omega_{1}}(\kappa)\right)$ where $T_{0}: \mathbb{C}(X)\left[u, u^{-1}\right] \rightarrow$ $\mathbb{C}(X)$ assigns to each Laurent polynomial in $u$ its constant term. By (6) $\operatorname{div}_{\omega_{1}}(\kappa)=$ $\operatorname{div}_{\omega_{0} \wedge d u}(\kappa)-\kappa(u) / u=\operatorname{div}_{\omega_{0} \wedge d u}(\kappa)-f_{0} / u$. Hence

$$
T_{0}\left(\operatorname{div}_{\omega_{1}}(\kappa)\right)=\operatorname{div}_{\omega_{0}}\left(\mu_{0}\right)+T_{0}\left(\partial f_{0} / \partial u\right)-T_{0}\left(f_{0} / u\right) .
$$

The desired conclusion follows now from the obvious fact that $T_{0}\left(\partial f_{0} / \partial u\right)=T_{0}\left(f_{0} / u\right)$.

3.3. Proposition. Let $C$ be the smooth zero locus of $p$ in $X$. Suppose also that the following conditions hold:

(A1) the linear space $\operatorname{VF}_{\text {alg }}^{\omega}(X, C)$ is generated by vector fields that are of the form $\widetilde{\operatorname{Pr}}\left(\left[\left[\mu_{f}, \nu_{1}^{\prime}\right], \nu_{2}^{\prime}\right]\right)$ where $\mu_{f}$ and $\nu_{i}^{\prime}$ are as in formula (9) from Lemma 2.5 with $\nu_{i} \in$ $\operatorname{IVF}_{\text {alg }}^{\omega}(X)$;

(A2) $\operatorname{VF}_{\text {alg }}(X)$ is generated by $\operatorname{IVF}_{\text {alg }}^{\omega}(X)$ as a module over $\mathbb{C}[X]$. 
Then $\mathrm{Lie}_{\text {alg }}^{\omega}\left(X^{\prime}\right)$ coincides with $\mathrm{VF}_{\text {alg }}^{\omega}\left(X^{\prime}\right)$, i.e., $X^{\prime}$ has the algebraic volume density property.

Proof. Let $\lambda, f_{0}$, and $g_{0}$ be as in formula (8) and $\Lambda=\iota(\lambda)$ be the extension of $\lambda$ to $Y$ also given by formula (8). By formula (7) $f_{0}$ and $g_{0}$ can be written uniquely in the form

$$
f_{0}=\sum_{i=1}^{m}\left(a_{i} u^{i}+b_{i} v^{i}\right)+a_{0} \text { and } g_{0}=\sum_{i=1}^{m}\left(\hat{a}_{i} u^{i}+\hat{b}_{i} v^{i}\right)+\hat{a}_{0}
$$

where $a_{i}, \hat{a}_{i}, b_{i}, \hat{b}_{i} \in \mathbb{C}[X]$.

Since $\Lambda$ is a vector field tangent to $X^{\prime}=P^{-1}(0)$ we have $\left.\Lambda(P)\right|_{X^{\prime}}=0$. Thus $0=\operatorname{Pr}\left(\iota\left(\left.\Lambda(P)\right|_{X^{\prime}}\right)\right)=p\left(a_{1}+\hat{b}_{1}\right)-\mu_{0}(p)$ (recall that $\left.P=u v-p(x)\right)$. Hence $\mu_{0}(p)$ vanishes on $C$, i.e. by Lemma $3.2 \mu_{0} \in \operatorname{VF}_{\text {alg }}^{\omega}(X, C)$. Let $\mu_{f}, \nu_{i}^{\prime} \in \operatorname{IVF}_{\text {alg }}^{\omega}\left(X^{\prime}\right)$ be as in Lemma 2.5. Condition (A1) implies that adding elements of the form $\left[\left[\mu_{f}, \nu_{1}^{\prime}\right], \nu_{2}^{\prime}\right]$ to $\lambda$ we can suppose that $\mu_{0}=0$. Using condition (A2) and Lemma 2.5 (iv) we can make $\mu_{i}^{j}=0$ by adding fields of the form $\left[\mu_{f}, \nu_{i}^{\prime}\right]$ with $\nu_{i} \in \operatorname{IVF}_{\text {alg }}^{\omega}(X)$. Note that this addition leaves not only $\mu_{0}$ equal to 0 but also $\operatorname{div}_{\omega^{\prime}}(\lambda)$ equal to 0 , since $\operatorname{div}_{\omega^{\prime}}\left(\left[\mu_{f}, \nu_{i}^{\prime}\right]\right)=0$ as soon as $\operatorname{div}_{\omega}\left(\nu_{i}\right)=0$. Hence $\lambda=f \partial / \partial u+g \partial / \partial v$ and $\left.\Lambda(P)\right|_{X^{\prime}}=f v+g u=0$.

Using formula (7) one can see that $f$ must be divisible by $u$, and $g$ by $v$. That is, there exists a regular function $h$ on $X^{\prime}$ for which $f=u h$ and $g=-v h$. Hence $\lambda=h(u \partial / \partial u-v \partial / \partial v)$. Note that $\Lambda(P)=0$ now. Thus by Lemma 2.1

$$
0=\operatorname{div}_{\omega^{\prime}}(\lambda)=\left.\operatorname{div}_{\Omega}(\Lambda)\right|_{X^{\prime}}=\left.\left(u \frac{\partial h}{\partial u}-v \frac{\partial h}{\partial v}\right)\right|_{X^{\prime}}
$$

Taking $h$ as in formula (7) we see that $h$ is independent of $u$ and $v$. Thus $\lambda$ is integrable and of zero divergence by Lemma 2.5 (ii)-(iii).

Taking commutative vector fields $\nu_{1}$ and $\nu_{2}$ from $\operatorname{IVF}_{\text {alg }}^{\omega}(X)$ in formula (9) we have the following.

3.4. Lemma. Condition (A1) in Proposition 3.3 holds if $\mathrm{VF}_{\mathrm{alg}}^{\omega}(X, C)$ is generated as a linear space by vector fields of the form $\nu_{1}(f p) \nu_{2}-\nu_{2}(f p) \nu_{1}$ where the vector fields $\nu_{1}, \nu_{2} \in \operatorname{IVF}_{\text {alg }}^{\omega}(X)$ are commutative.

It is more convenient for us to reformulate this new condition in terms of differential forms for which we need some extra facts. Let $\iota_{\nu}$ be the inner product with a vector field $\nu$ on $X$. Recall the following relations between the outer differentiation d, the Lie derivative $L_{\nu}$ and $\iota_{\nu}$

$$
L_{\nu}=\mathrm{d} \circ \iota_{\nu}+\iota_{\nu} \circ \mathrm{d} \text { and }\left[L_{\nu_{1}}, \iota_{\nu_{2}}\right]=\iota_{\left[\nu_{1}, \nu_{2}\right]} .
$$

Set $\omega_{\nu}=\iota_{\nu}(\omega)$. Then by formula (2) we have

$$
\operatorname{div}_{\omega}(\nu) \omega=\mathrm{d} \circ \iota_{\nu}(\omega)+\iota_{\nu} \circ \mathrm{d}(\omega)=\mathrm{d}\left(\omega_{\nu}\right) .
$$

Thus we have the first statement of the following. 


\subsection{Lemma.}

(1) A vector field $\nu$ is of zero divergence if and only if the form $\omega_{\nu}$ is closed.

(2) Furthermore, for a zero divergence field $\nu$ and every regular function $f$ on $X$ we have $\mathrm{d}\left(\omega_{f \nu}\right)=\nu(f) \omega$.

(3) Let $\nu_{1}, \nu_{2} \in \operatorname{IVF}_{\text {alg }}^{\omega}(X)$ be commutative and $\kappa=\nu_{1}(f p) \nu_{2}-\nu_{2}(f p) \nu_{1}$. Then $\mathrm{d}\left(\iota_{\nu_{1}} \circ \iota_{\nu_{2}}(f p \omega)\right)=\iota_{\kappa}(\omega)$ where $p, f \in \mathbb{C}[X]$.

Proof. Indeed, by (10)

$$
\mathrm{d}\left(\omega_{f \nu}\right)=L_{f \nu}(\omega)-\iota_{f \nu} \mathrm{d}(\omega)=L_{f \nu}(\omega)=\operatorname{div}_{\omega}(f \nu) \omega=\left(f \operatorname{div}_{\omega}(\nu)+\nu(f)\right) \omega=\nu(f) \omega
$$

which is (2).

Again by (10) we have

$$
\mathrm{d} \circ \iota_{\nu_{1}} \circ \iota_{\nu_{2}}(f p \omega)=L_{\nu_{1}} \circ \iota_{\nu_{2}}(f p \omega)-\iota_{\nu_{1}} \circ \mathrm{d} \circ \iota_{\nu_{2}}(f p \omega) .
$$

Then

$$
L_{\nu_{1}} \circ \iota_{\nu_{2}}(f p \omega)=L_{\nu_{1}}(f p) \iota_{\nu_{2}}(\omega)+f p L_{\nu_{1}} \circ \iota_{\nu_{2}}(\omega)
$$

and

$$
L_{\nu_{1}} \circ \iota_{\nu_{2}}(\omega)=\iota_{\nu_{2}} L_{\nu_{1}}(\omega)+\iota_{\left[\nu_{1}, \nu_{2}\right]}(\omega)=0
$$

since $\left[\nu_{1}, \nu_{2}\right]=0$ and $L_{\nu_{i}}(\omega)=0$. Similarly

$\left.\left.\iota_{\nu_{1}} \circ \mathrm{d} \circ \iota_{\nu_{2}}(f p \omega)\right)=L_{\nu_{2}}(f p) \iota_{\nu_{1}}(\omega)+f p \iota_{\nu_{1}} \circ L_{\nu_{2}}(\omega)-\iota_{\nu_{1}} \circ \iota_{\nu_{2}} \circ \mathrm{d}(f p \omega)\right)=L_{\nu_{2}}(f p) \iota_{\nu_{1}}(\omega)$.

Therefore,

$$
\mathrm{d} \circ \iota_{\nu_{1}} \circ \iota_{\nu_{2}}(f p \omega)=L_{\nu_{1}}(f p) \iota_{\nu_{2}}(\omega)-L_{\nu_{2}}(f p) \iota_{\nu_{1}}(\omega)=\nu_{1}(f p) \iota_{\nu_{2}}(\omega)-\nu_{2}(f p) \iota_{\nu_{1}}(\omega)
$$

which yields the desired conclusion.

Suppose that $\Omega^{q}(X)$ is the sheaf of algebraic $q$-forms on $X, \Omega_{i}^{q}(X)$ is its subsheaf that consists of forms that vanish on $C$ with multiplicity at least $i$ for $i \geq 1$, and vanish on all elements $\Lambda^{n-1} T C \subset \Lambda^{n-1} T X$ for $i=0$ where $\Lambda^{q} T X$ is the $q$-h wedge-power of $T X$, i.e. the set of $q$-dimensional subspaces of the tangent bundle. For every sheaf $\mathcal{F}$ on $X$ denote by $\Gamma^{0}(X, \mathcal{F})$ the space of global sections. That is, $\Gamma^{0}\left(X, \Omega_{1}^{n-2}(X)\right)$ is the subset of $\Gamma^{0}\left(X, \Omega^{n-2}(X)\right)$, that consists of forms divisible by $p$, and $\Gamma^{0}\left(X, \Omega_{0}^{n-1}(X)\right)$ is the set of algebraic $(n-1)$-forms on $X$ whose restriction to the zero fiber $C$ of $p$ yields a trivial form on $C$.

As a consequence Lemma 3.5 we have the following fact.

3.6. Lemma. Let $\kappa_{i}^{f}=\nu_{1}^{i}(f p) \nu_{2}^{i}-\nu_{2}^{i}(f p) \nu_{1}^{i}$ and let the following condition hold:

(B) there exists a collection $\left\{\nu_{1}^{i}, \nu_{2}^{i}\right\}_{i=1}^{m}$ of pairs of commutative vector fields from $\operatorname{IVF}_{\mathrm{alg}}^{\omega}(X)$ such that the set $\left\{\iota_{\nu_{1}^{i}} \circ \iota_{\nu_{2}^{i}}(\omega)\right\}_{i=1}^{m}$ generates the space of algebraic $(n-2)$ forms $\Gamma^{0}\left(X, \Omega^{n-2}(X)\right)$ on $X$ as $\mathbb{C}[X]$-module.

Then the image of $\Gamma^{0}\left(X, \Omega_{1}^{n-2}(X)\right)$ under the outer differentiation $\mathrm{d}: \Gamma^{0}\left(X, \Omega^{n-2}(X)\right) \rightarrow$ $\Gamma^{0}\left(X, \Omega^{n-1}(X)\right)$ is generated as a vector space by $(n-1)$-forms $\left\{\iota_{\kappa_{i}^{f}}(\omega)\right\}_{i=1}^{n}, f \in \mathbb{C}[X]$. 
3.7. Application of Grothendieck's theorem. Let $\mathcal{Z}^{0}\left(X, \Omega_{0}^{n-1}(X)\right)$ be the subspace of closed algebraic $(n-1)$-forms in $\Gamma^{0}\left(X, \Omega_{0}^{n-1}\right)$. Clearly, for every algebraic vector field $\nu \in \operatorname{IVF}_{\omega}(X)$ tangent to $C$ we have $\omega_{\nu} \in \mathcal{Z}^{0}\left(X, \Omega_{0}^{n-1}(X)\right)$. Our aim now is to show that under mild assumption the homomorphism

$$
\mathrm{d}: \Gamma^{0}\left(X, \Omega_{1}^{n-2}(X)\right) \rightarrow \mathcal{Z}^{0}\left(X, \Omega_{0}^{n-1}(X)\right)
$$

is surjective and, therefore, condition (A1) from Proposition 3.3 follows from condition (B) from Lemma 3.6. Denote by $\mathcal{F}_{i}^{\prime}$ (resp. $\mathcal{F}_{i}$ ) the space of algebraic sections of $\Omega_{n-1-i}^{i}$ (resp. $\Omega^{i}$ ) over $X$. Note that the outer differentiation d makes

$$
\mathcal{F}^{\prime}(*):=\ldots \rightarrow \mathcal{F}_{i}^{\prime} \rightarrow \mathcal{F}_{i+1}^{\prime} \rightarrow \ldots \text { and } \mathcal{F}(*):=\ldots \rightarrow \mathcal{F}_{i} \rightarrow \mathcal{F}_{i+1} \rightarrow \ldots
$$

complexes, and that the surjectivity we need for condition (B) would follow from $H^{n-1}\left(\mathcal{F}^{\prime}(*)\right)=0$.

3.8. Proposition. Let $H^{n-1}(X, \mathbb{C})=0$ and let the homomorphism $H^{n-2}(X, \mathbb{C}) \rightarrow$ $H^{n-2}(C, \mathbb{C})$ generated by the natural embedding $C \hookrightarrow X$ be surjective. Then $H^{n-1}\left(\mathcal{F}^{\prime}(*)\right)$ $=0$.

Proof. Consider the following short exact sequence of complexes $0 \rightarrow \mathcal{F}^{\prime}(*) \rightarrow \mathcal{F}(*) \rightarrow$ $\mathcal{F}^{\prime \prime}(*) \rightarrow 0$ where $\mathcal{F}_{i}^{\prime \prime}=\mathcal{F}_{i} / \mathcal{F}_{i}^{\prime}$ in complex $\mathcal{F}^{\prime \prime}(*)$. This implies the following long exact sequence in cohomology

$$
\ldots \rightarrow H^{n-2}(\mathcal{F}(*)) \rightarrow H^{n-2}\left(\mathcal{F}^{\prime \prime}(*)\right) \rightarrow H^{n-1}\left(\mathcal{F}^{\prime}(*)\right) \rightarrow H^{n-1}(\mathcal{F}(*)) \rightarrow \ldots,
$$

i.e. we need to show (i) that the homomorphism $H^{n-2}(\mathcal{F}(*)) \rightarrow H^{n-2}\left(\mathcal{F}^{\prime \prime}(*)\right)$ is surjective and (ii) that $H^{n-1}(\mathcal{F}(*))=0$. By the Grothendieck theorem [9] De Rham cohomology on smooth affine algebraic varieties can be computed via the complex of algebraic differential forms, i.e. $H^{n-1}(\mathcal{F}(*))=H^{n-1}(X, \mathbb{C})$ which implies (ii). Similarly, $H^{n-2}(\mathcal{F}(*))=H^{n-2}(X, \mathbb{C})$. Note that $\mathcal{F}_{i}^{\prime \prime}=\mathcal{F}_{i} /\left(p^{n-1-i} \mathcal{F}_{i}\right)$ for $i \leq n-2$. In particular, modulo the space $\mathcal{S}$ of (the restrictions to $C$ of) algebraic $(n-2)$-form that vanish on $\Lambda^{n-2} T C$ the term $\mathcal{F}_{n-2}^{\prime \prime}$ coincides with the space $\mathcal{T}$ of algebraic $(n-2)$-forms on $C$ (more precisely, we have the following exact sequence $0 \rightarrow \mathcal{S} \rightarrow \mathcal{F}_{n-2}^{\prime \prime} \rightarrow \mathcal{T} \rightarrow 0$ ). One can see that each closed $\tau \in \mathcal{S}$ is of form $\mathrm{d} p \wedge \tau_{0}$ where $\tau_{0}$ is a closed $(n-3)$-form on $C$. Hence $\tau=\mathrm{d}\left(p \tau_{0}\right)$ (where $p \tau_{0}$ can be viewed as an element of $\mathcal{F}_{n-3}^{\prime \prime}=\mathcal{F}_{n-3} /\left(p^{2} \mathcal{F}_{n-3}\right)$ ) is an exact form. Thus the $(n-2)$-cohomology of complex $\mathcal{F}^{\prime \prime}(*)$ coincides with the $(n-2)$-cohomology of the algebraic De Rham complex on $C$ and, therefore, is equal to $H^{n-2}(C, \mathbb{C})$ by the Grothendieck theorem. Now homomorphism from (i) becomes $H^{n-2}(X, \mathbb{C}) \rightarrow H^{n-2}(C, \mathbb{C})$ which implies the desired conclusion.

Thus we have Theorem 1 from Introduction as a consequence of the following more general fact (which gives, in particular, an affirmative answer to an open question of VAROLIN ([22], section 7) who asked whether the hypersurface $\left\{(a, b, c, d) \in \mathbb{C}^{4}\right.$ : $\left.a^{2} c-b d=1\right\}$ in $\mathbb{C}^{4}$ has the volume density property).

Theorem 3. Let $X$ be an $n$-dimensional smooth affine algebraic variety with $H^{n}(X, \mathbb{C})=$ 0 and a volume form $\omega$ satisfying conditions 
(B ) there exists a collection $\left\{\nu_{1}^{i}, \nu_{2}^{i}\right\}_{i=1}^{m}$ of pairs of commutative vector fields from $\operatorname{IVF}_{\mathrm{alg}}^{\omega}(X)$ such that the set $\left\{\iota_{\nu_{1}^{i}} \circ \iota_{\nu_{2}^{i}}(\omega)\right\}_{i=1}^{m}$ generates the space of algebraic $(n-2)$ forms $\Gamma^{0}\left(X, \Omega^{n-2}(X)\right)$ on $X$ as $\mathbb{C}[X]$-module;

(A2) $\mathrm{VF}_{\text {alg }}(X)$ is generated by $\operatorname{IVF}_{\text {alg }}^{\omega}(X)$ as a module over $\mathbb{C}[X]$.2

Suppose also that $p$ is a regular function on $X$ with a smooth reduced zero fiber $C$ such that the homomorphism $H^{n-2}(X, \mathbb{C}) \rightarrow H^{n-2}(C, \mathbb{C})$ generated by the natural embedding $C \hookrightarrow X$ is surjective. Let $X^{\prime} \subset X \times \mathbb{C}_{u, v}^{2}$ be the hypersurface given by $u v=p$ and let $\omega^{\prime}$ be the pullback of the form $\omega \wedge d u / u$ on $Z=X \times \mathbb{C}_{u}$ under the natural projection $X^{\prime} \rightarrow Z$ 3. Then $X^{\prime}$ has the algebraic $\omega^{\prime}$-density property.

3.9. Algebraic volume density for $S L_{2}(\mathbb{C})$ and $P S L_{2}(\mathbb{C})$. Since $X^{\prime}=S L_{2}(\mathbb{C})$ is isomorphic to the hypersurface $u v=x_{1} x_{2}+1=: p(\bar{x})$ in $Y=\mathbb{C}_{\bar{x}, u, v}^{4}=X \times \mathbb{C}_{u, v}^{2}$ with $\bar{x}=\left(x_{1}, x_{2}\right)$ and $X=\mathbb{C}_{\bar{x}}^{2}$, Theorem 1 implies that $S L_{2}(\mathbb{C})$ has the algebraic volume density property with respect to the volume form $\omega^{\prime}$ on $X^{\prime}$ such that $\omega^{\prime} \wedge \mathrm{d} P=\Omega$ where $P=u v-p(\bar{x})$ and $\Omega=\mathrm{d} x_{1} \wedge \mathrm{d} x_{2} \wedge \mathrm{d} u \wedge \mathrm{d} v$ is the standard volume form on $\mathbb{C}^{4}$. On the other hand by Remark 2.2 (1) we can consider forms $\left(\mathrm{d} x_{1} \wedge \mathrm{d} x_{2} \wedge \mathrm{d} u\right) / u$, $\left(\mathrm{d} x_{1} \wedge \mathrm{d} x_{2} \wedge \mathrm{d} v\right) / v$, etc.. Each of these forms coincides with $\omega^{\prime}$ up to a sign because their wedge-products with $\mathrm{d} P$ are $\pm \Omega$. Note that $\left(\mathrm{d} x_{1} \wedge \mathrm{d} x_{2} \wedge \mathrm{d} u\right) / u$ is invariant with respect to the $\mathbb{C}_{+}$-action on $S L_{2}(\mathbb{C})$ given by $\left(x_{1}, x_{2}, u, v\right) \rightarrow\left(x_{1}, x_{2}+t x_{1}, u, v+t u\right), t \in \mathbb{C}_{+}$ which is generated by multiplications of a $\mathbb{C}_{+}$-subgroup of $S L_{2}(\mathbb{C})$. Thus $\omega^{\prime}$ is invariant with respect to such multiplications. Similarly, consideration of $\left(\mathrm{d} x_{1} \wedge \mathrm{d} x_{2} \wedge \mathrm{d} v\right) / v$ yields invariance with respect to the $\mathbb{C}_{+}$-action $\left(x_{1}, x_{2}, u, v\right) \rightarrow\left(x_{1}+t x_{2}, x_{2}, u+t v, v\right)$, etc.. This implies that $\omega^{\prime}$ is invariant with respect to multiplication by any element of $S L_{2}(\mathbb{C})$ and we proved the following result, which is originally due to Varolin ([22], Theorem 2).

3.10. Proposition. Group $S L_{2}(\mathbb{C})$ has the algebraic volume density property with respect to the invariant volume form.

Furthermore, since the commutative vector fields $\nu_{1}=\partial / \partial x_{1}$ and $\nu_{2}=\partial / \partial x_{2}$ on $X=\mathbb{C}_{\bar{x}}^{2}$ satisfy condition (B) of Lemma 3.6 we see that any vector field $\mu_{0}$ tangent to the zero fiber $C$ of $p$ is of form $\nu_{1}(f p) \nu_{2}-\nu_{2}(f p) \nu_{1}$ where $f$ is a polynomial on $X$. This fact will used in the next unpleasant computation which is similar to the argument in Proposition 3.3 .

3.11. Proposition. Group $P S L_{2}(\mathbb{C})$ has the algebraic volume density property with respect to the invariant volume form.

Proof. Consider now $X^{\prime \prime}=X^{\prime} / \mathbb{Z}_{2} \simeq P S L_{2}(\mathbb{C})$ where the $\mathbb{Z}_{2}$-action on $X^{\prime}$ given by $(u, v, \bar{x}) \rightarrow(-u,-v,-\bar{x})$. Note that $\mathbb{C}\left[X^{\prime \prime}\right]$ can be viewed as the subring of $\mathbb{C}\left[X^{\prime}\right]$ generated by monomials of even degrees. Hence completely integrable vector fields of form

$$
\nu_{1}^{\prime}=u^{i+1} \partial / \partial x_{k}+u^{i} \frac{\partial p}{\partial x_{k}} \partial / \partial v \text { and } \nu_{2}^{\prime}=v^{i+1} \partial / \partial x_{j}+v^{i} \frac{\partial p}{\partial x_{j}} \partial / \partial u
$$

\footnotetext{
${ }^{2}$ Clearly, the standard volume form on $\mathbb{C}^{n}$ satisfies both these conditions.

${ }^{3}$ One can check that $\omega^{\prime} \wedge d P=\left.\omega \wedge d u \wedge d v\right|_{X^{\prime}}$ where $P=u v-p$.
} 
$\left(\operatorname{resp} . \quad \nu_{1}^{\prime \prime}=u^{i+1} x_{j} \partial / \partial x_{k}+u^{i} x_{j} \frac{\partial p}{\partial x_{k}} \partial / \partial v\right.$ and $\left.\nu_{2}^{\prime \prime}=v^{i+1} x_{k} \partial / \partial x_{j}+v^{i} x_{k} \frac{\partial p}{\partial x_{j}} \partial / \partial u\right)$ on $X^{\prime}$ with even (resp. odd) $i$ can viewed as fields on $X^{\prime \prime}$. The same is true for $\mu_{f}$ from Lemma 2.5 provided $f$ is a linear combination of monomials of even degrees. Fields $\nu_{1}^{\prime}, \nu_{2}^{\prime}, \mu_{f}$ are of zero divergence. If $j \neq k$ the same holds for $\nu_{1}^{\prime \prime}$ and $\nu_{2}^{\prime \prime}$. Any algebraic vector field $\lambda$ on $X^{\prime \prime}$ can be viewed as a vector field on $X^{\prime}$ and, therefore, it is given by formula (8). Since this field on $X^{\prime}$ came from $X^{\prime \prime}$ each $\tilde{\mu}_{i}^{k}$ (resp. $\tilde{\mu}_{0}$ ) in that formula consists of summands of form $q(\bar{x}) \partial / \partial x_{k}$ where polynomial $u^{i} q(\bar{x})$ (resp. $\left.v^{i} q(\bar{x})\right)$ is a linear combination of monomials of odd degrees. Our plan is to simplify the form of a zero divergence field $\lambda$ on $X^{\prime \prime}$ by adding elements of the Lie algebra generated by fields like $\mu_{f}, \nu_{1}^{\prime}, \nu_{2}^{\prime}, \nu_{1}^{\prime \prime}, \nu_{2}^{\prime \prime}$.

Recall that $\tilde{\mu}_{0}$ is generated by a field $\mu_{0}$ on $X$ and it was shown in the proof of Proposition 3.3 that $\mu_{0}$ is tangent to $C$. Hence, as we mentioned before $\mu_{0}=\nu_{1}(f p) \nu_{2}-$ $\nu_{2}(f p) \nu_{1}$. Furthermore, if $\lambda$ comes from a field on $X^{\prime \prime}$ polynomial $f \in \mathbb{C}\left[x_{1}, x_{2}\right]$ must contain monomials of even degrees only. Thus by virtue of Lemma 2.5 (v) adding to $\lambda$ vector fields of form $\left[\left[\mu_{f}, \nu_{1}^{\prime}\right], \nu_{2}^{\prime}\right]$ we can suppose that $\tilde{\mu}_{0}=0$ without changing the divergence of $\lambda$.

Following the pattern of the proof of Proposition 3.3 let us add to $\lambda$ the zero divergence fields of form $\left[\mu_{f}, \nu_{l}^{\prime}\right]$ and $\left[\mu_{f}, \nu_{l}^{\prime \prime}\right]$. Since we have to require that $j \neq k$ in the definition of $\nu_{1}^{\prime \prime}$ and $\nu_{2}^{\prime \prime}$ we cannot eliminate summands $u^{i+1} \tilde{\mu}_{i+1}^{1}$ and $v^{i+1} \tilde{\mu}_{i+1}^{2}$ completely. However, Lemma 2.5 (iv) shows that after such addition one can suppose that $u^{i+1} \tilde{\mu}_{i+1}^{1}$ vanishes for even $i$, and for odd $i$ it is a linear combination of terms of form $u^{i+1} x_{k}^{m} \partial / \partial x_{k}$ where $m$ is odd (and similarly for $v^{i+1} \tilde{\mu}_{i+1}^{2}$ ).

Consider the semi-simple vector field $\nu=x_{1} \partial / \partial x_{1}-x_{2} \partial / \partial x_{2}$ on $X$. Then $\nu^{\prime}=$ $u^{i+1} \tilde{\nu}+u^{i} \nu(p) \partial / \partial v$ is a completely integrable zero divergence vector field on $X^{\prime}$ and for odd $i$ it can be viewed as a field on $X^{\prime \prime}$. Set $f=x_{1}^{m-1}$. By Lemma 2.5 (iv)

$$
\left[\mu_{f}, \nu^{\prime}\right]=(i+1) u^{i+1} x_{1}^{m-1} \tilde{\nu}+\alpha \partial / \partial u+\beta \partial / \partial v
$$

Thus adding a multiple of $\left[\mu_{f}, \nu^{\prime}\right]$ to $\lambda$ we can replace terms $u^{i+1} x_{1}^{m} \partial / \partial x_{1}$ in $u^{i+1} \tilde{\mu}_{i+1}^{1}$ by $u^{i+1} x_{1}^{m-1} x_{2} \partial / \partial x_{2}$. If $m \geq 2$ the latter can be taken care of by adding fields of form $\left[\mu_{f}, \nu_{i}^{\prime \prime}\right]$. If $m=1$ we cannot eliminate immediately terms like $u^{i} x_{1} \partial / \partial x_{1}$ or $u^{i} x_{2} \partial / \partial x_{2}$, but adding fields of form $c u^{i} \tilde{\nu}$ where $c$ is a constant we can suppose that only one of these terms is present. The same is true for similar terms with $u$ replaced by $v$. Thus adding elements from $\operatorname{Lie}^{\omega}\left(X^{\prime \prime}\right)$ we can reduce $\lambda$ to a zero divergence field of the following form

$$
\lambda=\sum_{i \geq 1}\left(c_{i} u^{i} x_{1} \partial / \partial x_{1}+d_{i} v^{i} x_{2} \partial / \partial x_{2}\right)+g_{1} \partial / \partial u+g_{2} \partial / \partial v
$$

where constants $c_{i}$ and $d_{i}$ may be different from zero only for even indices $i$ and by formula (7) $g_{k}=\sum_{i \geq 1}\left(a_{i}^{k}(\bar{x}) u^{i}+b_{i}^{k}(\bar{x}) v^{i}\right)+a_{0}^{k}(\bar{x})$ with $a_{i}^{k}$ and $b_{i}^{k}$ being polynomials on $X$. Since divergence $\operatorname{div}_{\omega} \lambda=0$ we immediately have $a_{i+1}^{1}=-c_{i} /(i+1)$ and $b_{i+1}^{2}=-d_{i} /(i+1)$, i.e. these polynomials are constants. 
Consider now an automorphism of $X^{\prime \prime}$ (and, therefore, of $X^{\prime}$ ) given by $\left(u, v, x_{1}, x_{2}\right) \rightarrow$ $\left(-x_{1}, x_{2},-u, v\right)$, i.e. it exchanges the role of pairs $(u, v)$ and $\left(x_{1}, x_{2}\right)$. It transforms $\lambda$ into a field

$$
\sum_{i \geq 1}\left(a_{i+1}^{1} x_{1}^{i+1} \partial / \partial x_{1}+b_{i+1}^{2} x_{2}^{i+1} \partial / \partial x_{2}\right)+\lambda_{0}
$$

where $\lambda_{0}$ does not contain nonzero summands of form $a x_{1} \partial / \partial x_{1}$ (resp. $b x_{2} \partial / \partial x_{2}$ ) with $a$ (resp. $b$ ) being a regular function on $X^{\prime \prime}$ non-divisible by $x_{2}$ (resp. $x_{1}$ ). Hence adding fields of form $\left[\left[\mu_{f}, \nu_{1}^{\prime}\right], \nu_{2}^{\prime}\right],\left[\mu_{f}, \nu_{k}^{\prime}\right]$, and $\left[\mu_{f}, \nu_{k}^{\prime \prime}\right]$ as before we can suppose that $\tilde{\mu}_{0}$ and each $\tilde{\mu}_{i}^{1}$ and $\tilde{\mu}_{i}^{2}$ are equal to zero, i.e. $\lambda=e \partial / \partial u+g \partial / \partial v$. Furthermore, arguing as in Proposition 3.3 we see $\lambda=h(u \partial / \partial u-v \partial / \partial v)$ where $h$ is a polynomial on $X$, i.e. $\lambda$ is completely integrable. Since by construction it is a vector field on $X^{\prime \prime}$, we have proved that $X^{\prime \prime}$ possesses the algebraic volume density property.

\section{Two BAsic FACTS ABOUt THE ALGEBRAIC VOLUme DENSITY PROPERTY}

By considering differential forms and vector fields in local coordinate systems one can see that the map $\nu \rightarrow \omega_{\nu}:=\iota_{\nu}(\omega)$ is bijective and, therefore, establishes a duality between algebraic (resp. holomorphic) vector fields and the similar $(n-1)$-forms on $X$. This duality in combination with the Grothendieck theorem [9] enables us to prove another important fact.

4.1. Proposition. For an affine algebraic manifold $X$ equipped with an algebraic volume form $\omega$ the algebraic volume density property implies the volume density property (in the holomorphic sense).

Proof. We need to show that any holomorphic vector field $\mu$ such that $\mu(\omega)=0$ can be approximated by an algebraic vector field $\nu$ with $\nu(\omega)=0$. Since the form $\omega_{\mu}$ is closed, by the Grothendieck theorem one can find a closed algebraic $(n-1)$-form $\tau_{n-1}$ such that $\omega_{\mu}-\tau_{n-1}$ is exact, i.e. $\omega_{\mu}-\tau_{n-1}=\mathrm{d} \tau_{n-2}$ for some holomorphic $(n-2)$-form $\tau_{n-2}$. Then we can approximate $\tau_{n-2}$ by an algebraic $(n-2)$-form $\tau_{n-2}^{\prime}$. Hence the closed algebraic $(n-1)$-form $\tau_{n-1}+\mathrm{d} \tau_{n-2}^{\prime}$ yields an approximation of $\mu$. By duality $\tau_{n-1}+\mathrm{d} \tau_{n-2}^{\prime}$ is of form $\omega_{\nu}$ for some algebraic vector field $\nu$ (approximating $\mu$ and by Lemma 3.5 (1) $\nu$ is of zero $\omega$-divergence which is the desired conclusion.

4.2. Lemma. If $X$ has the algebraic volume density property, then there exist finitely many algebraic vector fields $\sigma_{1}, \ldots, \sigma_{m} \in \operatorname{Lie}_{\text {alg }}^{\omega}(X)$ that generate $\operatorname{VF}_{\text {alg }}(X)$ as a $\mathbb{C}[X]$ module.

Proof. Let $n=\operatorname{dim} X$. We start with the following.

Claim. The space of algebraic fields of zero divergence generates the tangent space of $X$ at each point.

Let $x \in X$ and $U$ be a Runge neighborhood of $x$ such that $H^{n-1}(U, \mathbb{C})=0$ (for example take a small sublevel set of a strictly plurisubharmonic exhaustion function 
on $X$ with minimum at $x$ ). Shrinking $U$ we can assume that in some holomorphic coordinate system $z_{1}, \ldots, z_{n}$ on $U$ the form $\left.\omega\right|_{U}$ is the standard volume $\mathrm{d} z_{1} \wedge \ldots \wedge \mathrm{d} z_{n}$. Thus the holomorphic vector fields $\partial / \partial z_{i}$ on $U$ are of zero divergence and they span the tangent space at $x$. We need to approximate them by global algebraic fields of zero divergence on $X$ which would yield our claim. For that let $\nu \in \operatorname{VF}_{h o l}^{\omega}(U)$. The inner product $\iota_{\nu}(\omega)=: \alpha$ is by Lemma 3.5 (1) a closed $(n-1)$-form on $U$ and since $H^{n-1}(U, \mathbb{C})=0$ we can find an $(n-2)$-form $\beta$ on $U$ with $\mathrm{d} \beta=\alpha$. Since $U$ is Runge in $X$ we can also approximate $\beta$ by a global algebraic $(n-2)$-form $\tilde{\beta}$ (uniformly on compacts in $U)$. Then the closed algebraic $(n-1)$-form $\mathrm{d} \tilde{\beta}$ approximates $\alpha$ and the unique algebraic vector field $\theta$ defined by $\iota_{\theta}(\omega)=\mathrm{d} \tilde{\beta}$ approximates $\nu$. Since $\mathrm{d} \tilde{\beta}$ is closed, the field $\theta$ is of zero divergence which concludes the proof of the Claim.

Now it follows from the Claim and the algebraic volume density property that there are $n$ vector fields in $\operatorname{Lie}_{\text {alg }}^{\omega}(X)$ which span the tangent space at a given point $x \in X$. By standard induction on the dimension, adding more fields to span the tangent spaces at points where it was not spanned yet, we get the assertion of the lemma.

4.3. Remark. The similar fact holds for the (holomorphic) density property, because the same argument implies the holomorphic version of the Claim. In fact, we can say more: completely integrable holomorphic fields of zero divergence on a Stein manifold $X$ with the density property generate the tangent space at each point. Indeed, since by the Claim such a tangent space is generated by elements of $\operatorname{Lie}_{\text {hol }}^{\omega}(X)$ it suffices to show that every Lie bracket $[\nu, \mu]$ of completely holomorphic integrable vector fields of zero divergence can be approximated by a linear combination of such fields which follows immediately from the equality $[\nu, \mu]=\lim _{t \rightarrow 0} \frac{\varphi_{t}^{*}(\nu)-\nu}{t}$ where $\varphi_{t}$ is the phase flow generated by $\mu$. The reason why we cannot make this stronger version of the Claim in the algebraic category is that for an algebraic vector field $\mu$ the phase flow $\varphi_{t}$ may be only holomorphic. Another interesting fact is that a set of completely integrable holomorphic zero divergence vector fields which is needed for generation of tangent spaces at each point of $X$ can be chosen finite.

Let us suppose that $X$ and $Y$ are affine algebraic manifolds equipped with volume forms $\omega_{X}$ and $\omega_{Y}$ respectively.

4.4. Proposition. Suppose that $X$ (resp. Y) has the algebraic $\omega_{X}$ (resp. $\omega_{Y}$ ) volume density property. Let $\omega=\omega_{X} \times \omega_{Y}$. Then $X \times Y$ has the algebraic volume density property relative to $\omega$.

Proof. By Lemma 4.2 we can suppose that $\sigma_{1}, \ldots, \sigma_{m} \in \operatorname{Lie}_{\text {alg }}^{\omega_{X}}(X)\left(\operatorname{resp} . \delta_{1}, \ldots, \delta_{n} \in\right.$ Lie $\left.\omega_{\text {alg }}^{\omega_{Y}}(Y)\right)$ generate $\operatorname{VF}_{\text {alg }}(X)$ as a $\mathbb{C}[X]$-module (resp. $\operatorname{VF}_{\text {alg }}(Y)$ as a $\mathbb{C}[Y]$-module).

Denote by $F_{Y}$ the vector subspace (over $\mathbb{C}$ ) of $\mathbb{C}[Y]$ generated by $\operatorname{Im} \delta_{1}, \ldots, \operatorname{Im} \delta_{n}$. Then $\mathbb{C}[Y]=F_{Y} \oplus V$ where $V$ is another subspace whose basis is $v_{1}, v_{2}, \ldots$ Set $F_{Y}^{\prime}=\mathbb{C}[X] \otimes F_{Y}$ and $V^{\prime}=\mathbb{C}[X] \otimes V$, i.e. the algebra of regular functions on $X \times Y$ is $A=\mathbb{C}[X] \otimes \mathbb{C}[Y]=F_{Y}^{\prime} \oplus V^{\prime}$. Let $f_{i} \in \mathbb{C}[X]$ and $g_{j} \in \mathbb{C}[Y]$. Note that $f_{i}$ is in 
the kernel of all globally integrable fields used in the Lie combination for $\delta_{i}$ and thus $f_{i} \delta_{i} \in \operatorname{Lie}_{\text {alg }}^{\omega_{Y}}(Y)$, analogously $g_{i} \sigma_{i} \in \operatorname{Lie}_{\text {alg }}^{\omega_{X}}(X)$. The fields $\delta_{i}$ and $\sigma_{j}$ generate (vertical and horizontal) vector fields on $X \times Y$ that are denoted by the same symbols. Consider

$$
\left[f_{i} \delta_{i}, g_{j} \sigma_{j}\right]=\delta_{i}\left(f_{i} g_{j}\right) \sigma_{j}-\sigma_{j}\left(f_{i} g_{j}\right) \delta_{i} .
$$

By construction $\delta_{i}$ and $\sigma_{j}$ are commutative and moreover $\operatorname{Span} f_{i} \cdot g_{i}=\mathbb{C}[X \times Y]$. Hence the coefficient before $\sigma_{j}$ runs over $\operatorname{Im} \delta_{i}$ and, therefore, for any $\alpha_{1}^{\prime}, \ldots, \alpha_{n}^{\prime} \in F_{Y}^{\prime}$ there are $\beta_{1}^{\prime}, \ldots, \beta_{m}^{\prime} \in A$ such that the vector field

$$
\sum_{j} \alpha_{j}^{\prime} \sigma_{j}-\sum_{i} \beta_{i}^{\prime} \delta_{i}
$$

belongs to $\operatorname{Lie}_{\text {alg }}^{\omega}(X \times Y)$. Thus adding vector fields of this form to a given vector field

$$
\nu=\sum_{j} \alpha_{j} \sigma_{j}-\sum_{i} \beta_{i} \delta_{i}
$$

from $\operatorname{VF}_{\text {alg }}^{\omega}(X \times Y)$ we can suppose that each $\alpha_{j} \in V^{\prime}$. Hence one can rewrite $\nu$ in the following form

$$
\nu=\sum_{l} \sum_{j}\left(h_{j l} \otimes v_{l}\right) \sigma_{j}-\sum_{i} \beta_{i} \delta_{i}
$$

where each $h_{j l} \in \mathbb{C}[X]$. Then one has

$$
0=\operatorname{div} \nu=\sum_{l}\left(\sum_{j} \sigma_{j}\left(h_{j l}\right)\right) \otimes v_{l}-\sum_{i} \delta_{i}\left(\beta_{i}\right) .
$$

Since the first summand is in $V^{\prime}$ and the last is in $F_{Y}^{\prime}$ we see that $\sum_{j} \sigma_{j}\left(h_{j l}\right)=0$, i.e. each vector field $\sum_{j} h_{j l} \sigma_{j}$ belong to $\operatorname{VF}_{\text {alg }}^{\omega_{X}}(X)$ and by the assumption to $\operatorname{Lie}_{\text {alg }}^{\omega_{X}}(X)$. Hence it suffices to prove the following

Claim. Consider the subspace $B \subset \mathrm{VF}_{\text {alg }}^{\omega}(X \times Y)$ that consists of vector fields of form

$$
\nu=\sum_{i} \beta_{i} \delta_{i}
$$

Then $B$ is contained in $\operatorname{Lie}_{\text {alg }}^{\omega}(X \times Y)$.

Indeed, consider a closed embedding of $Y$ into a Euclidean space. Then it generates filtration on $\mathbb{C}[Y]$ by minimal degrees of extensions of regular functions to polynomials. In turn this generates filtrations $B=\bigcup B_{i}$ and $\operatorname{Lie}_{a l g}^{\omega_{Y}}(X \times Y)=\bigcup L_{i}$. Note that each $B_{i}$ or $L_{i}$ is a finitely generated $\mathbb{C}[X]$-module, i.e. they generate coherent sheaves on $X$. Furthermore, since $Y$ has algebraic $\omega_{Y}$-density property we see that the quotients of $B_{i}$ and $L_{i}$ with respect to the maximal ideal corresponding to any point $x \in X$ coincide. Thus $B_{i}=L_{i}$ which implies the desired conclusion.

Note that up to a constant factor the completely integrable vector field $z \partial / \partial z$ on the group $X=\mathbb{C}^{*}$ is the only field of zero divergence with respect to the invariant volume form $\omega=\frac{\mathrm{d} z}{z}$, i.e., $X$ has the algebraic volume density property. Hence we have the following (see also Corollary 4.5 in [20]). 
4.5. Proposition. For every $n \geq 1$ the torus $\left(\mathbb{C}^{*}\right)^{n}$ has the algebraic volume density property with respect to the invariant form.

\section{Algebraic VOlume Density for locally trivial Fibrations}

5.1. Fine manifolds. If the subspace $V \simeq \mathbb{C}[Y] / F_{Y}$ in the proof of Proposition 4.4 were trivial so would be the proof but in the general case $V \neq 0$. We shall see later that Proposition 4.4 can be extended to some locally trivial fibrations with fiber $Y$ for which, in particular, $\mathbb{C}[Y] / F_{Y}$ is at most one-dimensional. Thus we need the following.

5.2. Definition. Let $Y$ be an affine algebraic manifold with a volume form $\omega$ and $F_{Y}$ be the subspace of $\mathbb{C}[Y]$ that consists of images of vector fields from $\operatorname{Lie}_{a l g}^{\omega}(Y)$. We say that $Y$ is a fine manifold if either $\mathbb{C}[Y]=F_{Y}$ or $\mathbb{C}[Y]$ is naturally isomorphic to $F_{Y} \oplus \mathbb{C}$ where the second summand denotes constant functions on $Y$.

5.3. Lemma. Let $Y$ be the smooth hypersurface in $\mathbb{C}_{u, v \bar{x}}^{n+2}$ given by $P=u v+q(\bar{x})-1=0$ where $q(\bar{x})=\sum_{i=1}^{n} x_{i}^{2}$ (i.e. after a coordinate change uv $+q(\bar{x})$ can be replaced by any non-degenerate quadratic form). Suppose that $Y$ is equipped with a volume $\omega_{Y}$ such that $\mathrm{d} P \wedge \omega=\Omega$ where $\Omega$ is the standard volume form on $\mathbb{C}^{n}$. Then

(1) $Y$ is a fine manifold;

(2) $Y / \mathbb{Z}_{2}$ is a fine manifold where the $\mathbb{Z}_{2}$-action is given by $(u, v, \bar{x}) \rightarrow(-u,-v,-\bar{x})$.

Proof. Consider the semi-simple vector field $\mu=u \partial / \partial u-v \partial / \partial v$ on $Y$. It generates $\mathbb{Z}$-grading of $\mathbb{C}[Y]=\oplus_{i \in \mathbb{Z}} A_{i}$ such that $\operatorname{Ker} \mu=A_{0}$ and $\operatorname{Im} \mu=\oplus_{i \in \mathbb{Z}, i \neq 0} A_{i} \subset F_{Y}$. Note that $A_{0} \simeq \mathbb{C}\left[x_{1}, \ldots, x_{n}\right]$ since $u v=1-q(x) \in A_{0}$. Assume for simplicity that $n \geq 2$ and replace $x_{1}$ and $x_{2}$ by $u^{\prime}=x_{1}+\sqrt{-1} x_{2}$ and $v^{\prime}=x_{1}-\sqrt{-1} x_{2}$ in our coordinate system. Consider the semi-simple vector field $\mu^{\prime}=u^{\prime} \partial / \partial u^{\prime}-v^{\prime} \partial / \partial v^{\prime}$ whose kernel is $A_{0}^{\prime}=\mathbb{C}\left[u, v, x_{3}, \ldots, x_{n}\right]$. Thus monomials containing $u^{\prime}$ and $v^{\prime}$ (or, equivalently, $x_{1}$ or $x_{2}$ in the original coordinate system) are in $\operatorname{Im} \mu^{\prime} \subset F_{Y}$. Repeating this procedure with other $x_{i}$ and $x_{j}$ instead of $x_{1}$ and $x_{2}$ we see that $F_{Y}$ contains every nonconstant monomial which is (1).

For (2) note that $\mathbb{C}\left[Y / \mathbb{Z}_{2}\right]$ is the subring of $\mathbb{C}[Y]$ generated by monomials of even degrees and that the semi-simple vector fields that we used preserve the standard degree function. That is, if a monomial $M_{1}$ of even degree belongs, say, to $\operatorname{Im} \mu$ then $M_{1}=\mu\left(M_{2}\right)$ where $M_{2}$ is also a monomial of even degree. This yields (2).

Since $S L_{2}(\mathbb{C})$ is isomorphic to the hypersurface $u v-x_{1} x_{2}=1$ in $\mathbb{C}_{u, v, x_{1}, x_{2}}^{4}$ we have the following.

5.4. Corollary. Both $S L_{2}(\mathbb{C})$ and $P S L_{2}(\mathbb{C})$ are fine manifolds.

5.5. Remark. In fact for $Y$ equal to $S L_{2}(\mathbb{C})$ or $P S L_{2}(\mathbb{C})$ we have $\mathbb{C}[Y] / F_{Y} \simeq \mathbb{C}$. More precisely, set $F=\operatorname{Span}\left\{\nu(f): \nu \in \mathrm{VF}_{\text {alg }}^{\omega}(Y), f \in \mathbb{C}[Y]\right\}$. Note that vector fields of form $f \nu$ span all algebraic vector fields because of Claim in Lemma 4.2 (and Lemma 5.9 below). Therefore, $(n-1)$-forms $\omega_{f \nu}$ generate all algebraic $(n-1)$-forms on $Y$ 
where $n=\operatorname{dim} Y$. By Lemma 3.5 $(2), \mathrm{d}\left(\omega_{f \nu}\right)=\nu(f) \omega$ which implies that the image of $\Omega^{n-1}(Y)$ in $\Omega^{n}(Y)$ under outer differentiation coincides with $F \omega$. Since $\mathrm{d}\left(\Omega^{n}(Y)\right)=0$ we have $\mathbb{C}[Y] / F \simeq H^{n}(Y, \mathbb{C})$ by the Grothendieck theorem. By Proposition 4.1 in [14] for a smooth hypersurface $Y \subset \mathbb{C}^{m+2}$ given by $u v=p(x)$ we have $H_{*}(Y)=H_{*-2}(C)$ where $C$ is the zero fiber of $p$. Thus the universal coefficient formula implies that $\operatorname{dim} \mathbb{C}[Y] / F=\operatorname{rank} H^{m-1}(C, \mathbb{C})$. For $S L_{2}(\mathbb{C})$ presented as such a hypersurface we have $p\left(x_{1}, x_{2}\right)=x_{1} x_{2}-1$, i.e. $C$ is a hyperbola and $H^{1}(C, \mathbb{C})=\mathbb{C}$ which yields the desired conclusion because $F=F_{Y}$ for manifolds with the algebraic volume density property.

5.6. Notation. Further in this section $X, Y$, and $W$ are smooth affine algebraic varieties and $p: W \rightarrow X$ is a locally trivial fibration with fiber $Y$ in the étale topology. We suppose also that $Y$ is equipped with a unique up to constant algebraic volume form $\omega_{Y}$, and $\mathrm{VF}_{\text {alg }}(W, p)$ (resp. $\mathrm{VF}_{\text {alg }}^{\omega_{Y}}(W, p)$ ) is the space of algebraic vector fields tangent to the fibers of $p$ (resp. and such that the restriction to each fiber has zero divergence relative to $\omega_{Y}$. ) Similarly $\operatorname{Lie}^{\omega_{Y}}(W, p)$ will be the Lie algebra generated by completely integrable vector fields from $\mathrm{VF}_{\text {alg }}^{\omega_{Y}}(W, p)$. We denote the subspace of $\mathbb{C}[W]$ generated by functions of form $\left\{\operatorname{Im} \nu \mid \nu \in \operatorname{Lie}^{\omega_{Y}}(W, p)\right\}$ by $F(W, p)$.

5.7. Definition. A family $\delta_{1}, \ldots, \delta_{n}, \ldots \in \operatorname{Lie}_{a l g}^{\omega_{Y}}(Y)$ will be called rich if $(1)$ it generates $\mathrm{Lie}_{\text {alg }}^{\omega_{Y}}(Y)$ as a Lie algebra and $(2) \mathrm{VF}_{\text {alg }}(Y)$ as a $\mathbb{C}[Y]$-module.

5.8. Remark. (i) Note that (1) implies the sets $\left\{\delta_{i}(\mathbb{C}[Y])\right\}$ generate the vector space $F_{Y}$.

(ii) For (2) it suffices to require that the set of vector fields $\delta_{1}, \ldots, \delta_{n}, \ldots$ generates the tangent space at each point of $Y$. This is a consequence of the next simple fact (e.g., see Exercise 5.8 in [10]) which is essentially the Nakayama lemma.

5.9. Lemma. Let $A \subset B$ be a finitely generated $\mathbb{C}[X]$-module and its submodule. Suppose that for every point $x \in X$ one has $A / M_{x}=B / M_{x}$ where $M_{x}$ is the maximal ideal in $\mathbb{C}[X]$ associated with $x$. Then $A=B$

5.10. Example. Let $\sigma_{1}, \sigma_{2}, \ldots\left(\right.$ resp. $\left.\delta_{1}, \delta_{2}, \ldots\right)$ be a a rich family on $X$ with respect to volume $\omega_{X}$ (resp. on $Y$ with respect to volume $\omega_{Y}$ ). Denote their natural lifts to $X \times Y$ by the same symbols. Consider the set $\mathcal{S}$ of "horizontal" and "vertical" fields of form $f \sigma_{i}$ and $g \delta_{j}$ where $f$ (resp. $g$ ) is a lift of a function on $Y$ (resp. $X$ ) to $X \times Y$. It follows from the explicit construction in the proof of Proposition 4.4 that $\mathcal{S}$ generates the Lie algebra $\operatorname{Lie}_{\omega}(X \times Y)$ for $\omega=\omega_{X} \times \omega_{Y}$, i.e. it satisfies condition (1) of Definition 5.7. Remark 5.8 (2) implies that condition (2) also holds and, therefore, $\mathcal{S}$ is a rich family on $X \times Y$.

In particular, consider a torus $\mathbb{T}=\left(\mathbb{C}^{*}\right)^{n}$ with coordinates $z_{1}, \ldots, z_{n}$. One can see that the vector field $\nu_{j}=z_{j} \partial / \partial z_{j}$ is a rich family on the $j$-th factor with respect to the invariant volume on $\mathbb{C}^{*}$. Thus fields of form $f_{j} \nu_{j}(j=1, \ldots, n)$ with $f_{j}$ being independent of $z_{j}$ generate a rich family on $\mathbb{T}$ with respect to the invariant volume. 
5.11. Convention. Furthermore, we suppose that vector fields $\delta_{1}, \ldots, \delta_{n}, \ldots$ form a rich family $\mathcal{S}$ in $\operatorname{Lie}_{a l g}^{\omega_{Y}}(Y)$ and there are vector fields $\delta_{1}^{\prime}, \ldots, \delta_{l}^{\prime}, \ldots \in \operatorname{VF}_{\text {alg }}^{\omega_{Y}}(W, p)$ such that up to nonzero constant factors the set of their restrictions to any fiber of $p$ contains $\delta_{1}, \ldots, \delta_{n}, \ldots$ under some isomorphism between this fiber and $Y$ ]

\subsection{Lemma.}

(1) A function $g \in \mathbb{C}[W]$ is contained in $F(W, p)$ if and only if its restriction to each general fiber of $p$ belongs to $F_{Y}$. Furthermore, if $Y$ is fine then $\mathbb{C}[W] \simeq F(W, p) \oplus \mathbb{C}[X]$.

(2) Suppose that $Y$ has the algebraic volume density property. Then $\operatorname{VF}_{a l g}^{\omega_{Y}}(W, p)=\operatorname{Lie}^{\omega_{Y}}(W, p)$.

Proof. There exists a cover $X=\bigcup_{i} X_{i}$ such that for each $i$ one can find an étale surjective morphism $X_{i}^{\prime} \rightarrow X_{i}$ for which the variety $W_{i}^{\prime}:=W_{i} \times_{X_{i}} X_{i}^{\prime}$ is naturally isomorphic to $X_{i}^{\prime} \times Y$ where $W_{i}=p^{-1}\left(X_{i}\right)$. Lifting functions on $W$ to some $W_{i_{0}}^{\prime}$ which is the direct product we can introduce filtration $\mathbb{C}[W]=\bigcup_{i \geq 0} G_{i}$ as we did in the Claim in Proposition 4.4 (i.e. take a closed embedding $Y \hookrightarrow \mathbb{C}^{m}$ and consider the minimal degrees of extensions of function on $W_{i_{0}}^{\prime}$ to $X_{i_{0}}^{\prime} \times \mathbb{C}^{m}$ with respect to the second factor).

Consider now the set $S$ of functions $g \in \mathbb{C}[W]$ such that for a general $x \in X$ the restriction $\left.g\right|_{p^{-1}(x)}$ is in $F_{Y}$. Since the degree (generated by the embedding $Y \hookrightarrow \mathbb{C}^{m}$ ) of the restriction of $g$ to each (not necessarily general) fiber $p^{-1}\left(x_{0}\right) \simeq Y$ is bounded by the same constant we see that $\left.g\right|_{p^{-1}\left(x_{0}\right)}$ belongs to $F_{Y}$ (because the finite-dimensional subspace of $F_{Y}$ that consists its elements, whose degrees are bounded by this constant, is closed). Set $S_{i}=S \cap G_{i}$ and $F_{i}=F(W, p) \cap G_{i}$. If suffices to show that $S_{i}=F_{i}$ for every $i$. Note that both $S_{i}$ and $F_{i}$ are finitely generated $\mathbb{C}[X]$-modules and because of the existence of fields $\delta_{1}^{\prime}, \ldots, \delta_{l}^{\prime}, \ldots$ in Convention 5.11 we see that for every $x \in X$ there is an equality $S_{i} / M_{x}=F_{i} / M_{x}$ where $M_{x}$ is the maximal ideal in $\mathbb{C}[X]$ associated with $x$. Hence the first statment of (1) follows from Lemma 5.9.

For the second statement of (1) note that $G_{i} / M_{x}=\left(F_{i} \oplus \mathbb{C}[X]\right) / M_{x}$ since $Y$ is fine and $\mathbb{C}[X] / M_{x}=\mathbb{C}$. Thus another application of Lemma 5.9] implies the desired conclusion.

The filtration of functions that we introduced, yields a filtrations of vector fields $\mathrm{VF}_{a l g}^{\omega_{Y}}(W, p)=\bigcup B_{i}$ and $\operatorname{Lie}^{\omega_{Y}}(W, p)=\bigcup L_{i}$ as where $B_{i}$ and $L_{i}$ are again finitely generated $\mathbb{C}[X]$-modules. Because $\mathcal{S}$ in Convention 5.11 is rich and $Y$ has the algebraic volume density property we have $B_{i} / M_{x}=L_{i} / M_{x}$. Thus by Lemma $5.9 B_{i}=L_{i}$ which yields $(2)$.

5.13. Definition. (1) Suppose that Convention 5.11 holds, $\omega_{X}$ is a volume form on $X$, $X=\bigcup X_{i}, X_{i}^{\prime}, W_{i}$, and $W_{i}^{\prime}$ are as in the proof of Lemma [5.12, $\varphi_{i}: W_{i}^{\prime} \rightarrow X_{i}^{\prime} \times Y$ is the natural isomorphism and $\omega$ is an algebraic volume form on $W$ such that up to a constant factor $\varphi_{i}^{*} \omega$ coincides with $\left(\left.\left(\omega_{X} \times \omega_{Y}\right)\right|_{X_{i} \times Y}\right)$ for each $i$. Then we call $p: W \rightarrow X$ a volume fibration (with respect to the volume forms $\omega_{X}, \omega_{Y}$, and $\omega$ ).

\footnotetext{
${ }^{4}$ If $p: W \rightarrow X$ is a Zariski locally trivial fibration this Convention is automatically true.

${ }^{5}$ In fact for $(1)$ one needs only that the sets $\left\{\delta_{i}(\mathbb{C}[Y])\right\}$ generate the vector space $F_{Y}$ with $\delta_{i}$ running over the family $\mathcal{S}$ from Convention 5.11 .
} 
(2) We call a derivation $\sigma^{\prime} \in \operatorname{Lie}^{\omega}(W)$ a lift of a derivation $\sigma \in \operatorname{Lie}^{\omega_{X}}(X)$ if for every $w \in W$ and $x=p(w)$ one has $p_{*}\left(\sigma^{\prime}(w)\right)=\sigma(x)$. (Note that the Lie bracket of two lifts is a lift.) We say that $\sigma^{\prime}$ is $p$-compatible if for any $\delta^{\prime} \in \mathrm{VF}_{\text {alg }}^{\omega}(W, p)$ we have $\left[\sigma^{\prime}, \delta^{\prime}\right] \in \operatorname{VF}_{a l g}^{\omega}(W, p)$ and the span $\operatorname{Span}\left(\operatorname{Ker} \sigma^{\prime} \cdot \operatorname{Ker} \delta^{\prime}\right)$ coincides with $\mathbb{C}[W]$.

(3) We call a volume fibration $p: W \rightarrow X$ refined if the fiber $Y$ is a fine manifold with algebraic volume density property, and a rich family of fields on $X$ has $p$-compatible lifts.

We shall see later (Lemma 6.11) that for a reductive group $G$ and its Levi semisimple subgroup $L$ the natural morphism $G \rightarrow G / L$ can be viewed as a refined volume fibration with respect to appropriate volume forms.

Since any algebraic vector field tangent to fiber of $p: W \rightarrow X$ has zero $\omega$-divergence if and only if its restriction on each fiber has zero $\omega_{Y}$-divergence we have the following consequence of Lemma $5.12(2)$.

5.14. Corollary. Let $p: W \rightarrow X$ be a volume fibration whose fiber has the algebraic volume density property and $\mathrm{VF}_{\text {alg }}^{\omega}(W, p)$ (resp. $\mathrm{Lie}^{\omega}(W, p)$ ) be the space of zero $\omega$-divergence algebraic vector fields tangent to the fibers of $p$ (resp. the Lie algebra generated by completely integrable algebraic vector fields tangent to the fibers of $p$ and of zero $\omega$-divergence). Then $\mathrm{VF}_{\text {alg }}^{\omega}(W, p)=\operatorname{Lie}^{\omega}(W, p)$.

The next fact will not be used further but it is interesting by itself.

5.15. Proposition. Let $p: W \rightarrow X$ be a refined volume fibration with a fine fiber $Y$ and fine base $X$. Then $W$ is fine.

Proof. Indeed, the existence of lifts in Definition 5.13 of a rich family makes $F_{X}$ and, therefore, $F(W, p) \oplus F_{X}$ a natural subspace of $F_{W}$. It remains to note that $\mathbb{C}[W]=$ $F(W, p) \oplus F_{X} \oplus \mathbb{C}$ by the assumption and by Lemma 5.12 .

5.16. Proposition. Let $p: W \rightarrow X$ be a refined volume fibration and $\Theta$ be the set of p-compatible lifts of a rich family from $\mathrm{Lie}_{\text {alg }}^{\omega_{X}}(X)$. Consider the space $L$ generated by $\operatorname{Lie}^{\omega}(W, p)$ and vector fields of form $\nu:=\left[f \sigma^{\prime}, \delta^{\prime}\right]$ where $\sigma^{\prime} \in \Theta, \delta^{\prime} \in \operatorname{Lie}^{\omega}(W, p)$, and $f \in \operatorname{Ker} \sigma^{\prime}$. Suppose that $T=p^{*}(T X)$ is the pull-back of the tangent bundle $T X$ to $W$, $\varrho: T W \rightarrow T$ is the natural projection, and $\mathcal{L}=\varrho(L)$. Then

(1) $\mathcal{L}$ is a $\mathbb{C}[X]$-module;

(2) $\mathcal{L}$ consists of all finite sums $\sum_{\sigma^{\prime} \in \Theta} h_{\sigma^{\prime}} \varrho\left(\sigma^{\prime}\right)$ where $h_{\sigma^{\prime}} \in F(W, p)$.

Proof. The space $\operatorname{Lie}^{\omega}(W, p)$ is, of course, a $\mathbb{C}[X]$-module. Thus it suffices to consider fields like $\nu=\left[\sigma^{\prime}, \delta^{\prime}\right]$ only. Since $\sigma^{\prime}$ is a lift of $\sigma \in \operatorname{Lie}^{\omega_{X}}(X)$ we see that $\sigma^{\prime}(\mathbb{C}[X]) \subset \mathbb{C}[X]$ where we treat $\mathbb{C}[X]$ in this formula as a subring of $\mathbb{C}[W]$. Then for every $\alpha \in \mathbb{C}[X]$ we have $\alpha \nu=\left[\sigma^{\prime}, \alpha \delta^{\prime}\right]-\sigma^{\prime}(\alpha) \delta^{\prime}$ which implies (1).

Let $f_{1} \in \operatorname{Ker} \sigma^{\prime}, f_{2} \in \operatorname{Ker} \delta^{\prime}$, and $h=\delta^{\prime}\left(f_{1} f_{2}\right)$. Then by the $p$-compatibility assumption $\left[f_{1} \sigma^{\prime}, f_{2} \delta^{\prime}\right]=h \sigma^{\prime}+a$ with $a \in \mathrm{VF}_{\text {alg }}(W, p)$ and, furthermore, the span of functions like $h$ coincides with $\delta^{\prime}(\mathbb{C}[W])$. Thus $\mathcal{L}$ contains $F(W, p) \varrho\left(\sigma^{\prime}\right)$ which is $(2)$. 
Theorem 4. Let $p: W \rightarrow X$ be a refined volume fibration whose fiber and base have the algebraic volume density property. Then $W$ has the algebraic volume density property.

Proof. Suppose that $\delta_{i}^{\prime}$ is as in Convention [5.11, $\left\{\sigma_{i}\right\}$ is a rich family on $X$, and $\sigma_{i}^{\prime} \in$ $\operatorname{Lie}^{\omega}(W)$ is a $p$-compatible lift of $\sigma_{i}$. Let $\kappa \in \mathrm{VF}_{\text {alg }}^{\omega}(W)$. Then $\kappa=\sum_{i} h_{i} \sigma_{i}^{\prime}+\theta$ where $h_{i} \in \mathbb{C}[W]$ and $\theta \in \mathrm{VF}_{\text {alg }}(W, p)$. By Proposition 5.16 and Lemma 5.12(1) adding an element of $L$ to $\kappa$ one can suppose that each $h_{i} \in \mathbb{C}[X]$. Since $\theta$ is a $\mathbb{C}[W]$-combination of $\delta_{1}^{\prime}, \ldots, \delta_{l}^{\prime}, \ldots$ and $\operatorname{div}_{\omega}\left(f \delta_{i}^{\prime}\right)=\delta_{i}^{\prime}(f)$, we see that $\operatorname{div}_{\omega} \theta \in F(W, p)$. On the other hand $\operatorname{div}_{\omega} \sum_{i} h_{i} \sigma_{i}^{\prime}=\sum_{i} \sigma_{i}\left(h_{i}\right)=\operatorname{div}_{\omega_{X}} \sum_{i} h_{i} \sigma_{i} \in \mathbb{C}[X]$. Hence $\operatorname{div}_{\omega_{X}} \sum_{i} h_{i} \sigma_{i}=0$ and $\theta \in \mathrm{VF}_{\text {alg }}^{\omega}(W, p)$. By assumption $\sum_{i} h_{i} \sigma_{i} \in L i e_{\text {alg }}^{\omega_{X}}(X)$. In combination with the existence of lifts for $\sigma_{i}$ and Corollary 5.14 this implies the desired conclusion.

\section{VOLUME FORMS ON HOMOGENEOUS SPACES}

6.1. Definition. We say that an affine algebraic variety $X$ is (weakly) rationally connected if for any (resp. general) points $x, y \in X$ there are a sequence of points $x_{0}=x, x_{1}, x_{2}, \ldots, x_{n}=y$ and a sequence of polynomial curves $C_{1}, \ldots, C_{n}$ in $X$ such that $x_{i-1}, x_{i} \in C_{i}$.

6.2. Remark. Since finite morphisms transforms polynomial curves into polynomial curves we have the following: if $X$ is an affine (weakly) rationally connected variety and $f: X \rightarrow Y$ is a finite morphism then $Y$ is also an affine (weakly) rationally connected variety.

6.3. Example. It is easy to see that $S L_{2}(\mathbb{C})$ is affine rationally connected. (Indeed, $S L_{2}(\mathbb{C})$ can be presented as an algebraic locally trivial $\mathbb{C}$-fibration over $\mathbb{C}^{2}$ without the origin $o$. Over any line in $\mathbb{C}^{2}$ that does not contain $o$ this fibration is trivial and, therefore, admits sections which implies the desired conclusion.) Hence any semisimple group is rationally connected since its simply connected covering is generated by $S L_{2}(\mathbb{C})$-subgroups.

6.4. Proposition. Let $X$ be an affine manifold and $\omega, \omega_{1}$ be algebraic volume forms on $X$.

(1) If $X$ is weakly rationally connected then $\omega=c \omega_{1}$ for nonzero constant $c$.

(2) If $G$ is a rationally connected linear algebraic group (say, unipotent or semisimple) acting on $X$ then $\omega \circ \Phi_{g}=\omega$ for the action $\Phi_{g}: X \rightarrow X$ of any element $g \in G$.

Proof. (1) Note that $\omega=h \omega_{1}$ where $h$ is an invertible regular function on $X$. Let $x, y, x_{i}, C_{i}$ be as in Definition 6.1. By the fundamental theorem of algebra $h$ must be constant on each $C_{i}$. Hence $h(x)=h(y)$ which implies the first statement.

(2) Let $e$ be the identity in $G$. Then we have a sequence $g_{0}=e, g_{1}, g_{2}, \ldots, g_{m}=g$ in $g$ such that for any $i \geq 1$ there is a polynomial curve $C_{i}$ in $G$ joining $g_{i-1}$ and $g_{i}$. Again for every $a \in C_{i}$ we have $\omega \circ \Phi_{a}=h(a) \omega$ where $h$ is a nonvanishing regular function on $C_{i}$, i.e. a constant. This implies $\omega \circ \Phi_{g}=\omega \circ \Phi_{e}=\omega$ which concludes the proof. 
For a Lie group $G$ one can construct a left-invariant (resp. right-invariant) algebraic volume form by spreading the volume element at identity by left (resp. right) multiplication (one of these forms can be transformed into the other by the automorphism $\varphi: G \rightarrow G$ given by $\varphi(g)=g^{-1}$ ). Proposition 6.4 yields now the following well-known facts.

6.5. Corollary. For a semi-simple Lie group $G$ its left-invariant volume form is automatically also right-invariant.

6.6. Remark. Since up to a finite covering any reductive group $G$ is a product of a torus and a semi-simple group we see that the left-invariant volume form on this group is also right-invariant.

6.7. Proposition. Let $W$ be a linear algebraic group group, $Y$ be its rationally connected subgroup, and $X=W / Y$ be the homogeneous space of left cosets. Then there exists an algebraic volume form $\omega_{X}$ on $X$ invariant under the action of $W$ generated by left multiplication.

Proof. Consider a left-invariant volume form $\omega$ on $W$ and left-invariant vector fields $\nu_{1}, \ldots, \nu_{m}$ on the coset $e Y \simeq Y$, where $e$ is the identity of $W$, so that they generate basis of the tangent space at any point of this coset. Extend these vector fields to $W$ using left multiplication. Since $e Y$ is a fiber of the natural projection $p: W \rightarrow X$ and left multiplication preserves the fibers of $p$ we see that the extended fields are tangent to all fibers of $p$. Consider the left-invariant form $\omega_{X}=\iota_{\nu_{1}} \circ \ldots \circ \iota_{\nu_{m}}(\omega)$ (where $\iota_{\nu_{i}}$ is the inner product of vector fields and differential forms). By construction it can be viewed as a non-vanishing form on vectors from the pull-back of the tangent bundle $T X$ to $W$. To see that it is actually a volume form on $X$ we have to show that it is invariant under right multiplication by any element $y \in Y$. Such multiplication generates an automorphism of $T W$ that sends vectors tangent (and, therefore, transversal) to fibers of $p$ to similar vectors. Hence it transforms $\omega_{X}$ into $f_{y} \omega_{X}$ where $y \rightarrow f_{y}$ is an algebraic homomorphism from $Y$ into the group of non-vanishing regular functions of $W$. Since the rationally connected group $Y$ has no nontrivial algebraic homomorphisms into $\mathbb{C}^{*}$ we have $f_{y} \equiv 1$ which yields the desired conclusion.

By Mostow's theorem [16] a linear algebraic group $W$ contains a Levi reductive subgroup $X$ such that as an affine algebraic variety $W$ is isomorphic to $X \times Y$ where $Y$ is the unipotent radical of $W$. More precisely, each element $w \in W$ can be uniquely presented as $w=g \cdot r$ where $g \in X$ and $r \in Y$. This presentation allows us to choose this isomorphism $W \rightarrow X \times Y$ uniquely.

6.8. Corollary. For the isomorphism $W \rightarrow X \times Y$ as before the left invariant volume form $\omega$ on $W$ coincides with $\omega_{X} \times \omega_{Y}$ where $\omega_{X}$ is a left-invariant volume form on $X$ and $\omega_{Y}$ is an invariant form on $Y$.

Proof. Note that $\omega$ is invariant by left multiplications (in particular by elements of $X$ ) and also by right multiplication by elements of $Y$ (see, Lemma 6.4(2)). This determines 
$\omega$ uniquely up to a constant factor. Similarly, by construction $\omega_{X} \times \omega_{Y}$ is invariant by left multiplications by elements of $X$ and by right multiplication by elements of $Y$.

6.9. Example. Consider the group $W$ of affine automorphisms $z \rightarrow a z+b$ of the complex line $\mathbb{C}$ with coordinate $z$. Then $Y \simeq \mathbb{C}_{+}$is the group of translations $z \rightarrow z+b$ and we can choose $X \simeq \mathbb{C}^{*}$ so that its elements are automorphisms of form $z \rightarrow a z$. One can check that the left-invariant volume $\omega$ on $W$ coincides with $\frac{\mathrm{d} a}{a^{2}} \wedge \mathrm{d} b$ while $\omega_{X}=\mathrm{d} a / a$ and $\omega_{Y}=\mathrm{d} b$. The isomorphism $W \rightarrow X \times Y$ we were talking about presents $a z+b$ as a composition of $a z$ and $z+b / a$. Thus in this case Corollary 6.8 boils down to the equality

$$
\frac{\mathrm{d} a}{a^{2}} \wedge \mathrm{d} b=\frac{\mathrm{d} a}{a} \wedge \frac{\mathrm{d} b}{a} .
$$

6.10. Proposition. Let $W$ be a linear algebraic group group, $Y$ be its rationally connected subgroup, and $X=W / Y$ be the homogeneous space of left cosets. Suppose that $\omega$ is the left-invariant volume form on $W, \omega_{Y}$ the invariant volume form on $Y$ and $\omega_{X}$ the volume form on the quotient constructed in Proposition 6.7. Then the natural projection $p: W \rightarrow X$ is a volume fibration with respect to the volume forms $\omega, \omega_{X}$, and $\omega_{Y}$.

Proof. Choose locally nilpotent derivations $\sigma_{1}^{\prime}, \ldots, \sigma_{k}^{\prime}$ and semi-simple derivations $\sigma_{k+1}^{\prime}$, $\ldots, \sigma_{n}^{\prime}$ on $W$ generated by the left multiplication of $W$ by elements of its $\mathbb{C}_{+}$and $\mathbb{C}^{*}$ subgroups and such that they generate tangent space at each point of $W$. Since they commute with morphism $p$ they yield locally nilpotent and semi-simple derivations $\sigma_{1}, \ldots, \sigma_{n}$ on $X$ with the same property. Take any point $x \in X$ and suppose that $\sigma_{l}, \ldots, \sigma_{m}$ generate the tangent space $T_{x} X$ (where $l \leq k \leq m$ ). Then we have the dominant natural morphism $\psi: G \rightarrow X$ from the group $G:=\mathbb{C}^{k-l+1} \times\left(\mathbb{C}^{*}\right)^{m-k}$ given by the formula $\bar{t}=\left(t_{l}, \ldots, t_{m}\right) \rightarrow h_{t_{l}} \circ \cdots \circ h_{t_{m}}(x)$ where $\bar{t} \in G$ and $h_{t_{j}}$ is the action of the element $t_{j}$ from the $\mathbb{C}_{+}$or $\mathbb{C}^{*}$-group corresponding to the $j$-th factor in $G$. This morphism is étale at the identical element $o=(0, \ldots, 0,1, \ldots, 1)$ of $G$ and $\psi(o)=x$. The restriction of $\psi$ to an open Zariski dense subvariety $Z$ of $\mathbb{C}^{m-l+1}$ may be viewed as an étale neighborhood of $x \in X$. Suppose that $\omega_{Z}$ (resp. $\left.\tilde{\sigma}_{i}\right)$ is the lift of the form $\omega_{X}$ (resp. vector field $\sigma_{i}$ ) to $Z$. By Proposition 6.7 $\omega_{Z}$ is invariant under the local phase flow generated by $\tilde{\sigma}_{i}$. Set $W^{\prime}=W \times_{X} Z$. Then by construction, $W^{\prime}$ is naturally isomorphic to $Z \times Y$ and under this isomorphism each field $\sigma_{i}^{\prime}$ corresponds to the horizontal lift of $\tilde{\sigma}_{i}$ to $Z \times Y$. Hence $\omega_{Z} \times \omega_{Y}$ is invariant under the local phase flow generated by this lift of $\tilde{\sigma}_{i}$. It is also invariant under right multiplication by elements of $Y$ by Proposition 6.4 (2) and, therefore, determined uniquely by its value at one point. But the form $\omega$ is also invariant under the local phase flow generated by $\sigma_{i}^{\prime}$ and under right multiplication by elements of $Y$ again by Proposition 6.4 (2). Therefore, the preimage of $\omega$ on $W$ coincides with $\omega_{Z} \times \omega_{Y}$ since one can choose $\omega_{Y}$ so that both forms coincide at one point. 
We finish this section with the following example of a refined volume fibrations.

6.11. Lemma. Let $W$ be a reductive group and $Y$ be its Levi semi-simple subgroup. Then $p: W \rightarrow X:=W / Y$ is a refined volume fibration.

Proof. By Proposition 6.10 it is a volume fibration. Thus by Definition 5.13 it remains to find a rich family of algebraic fields on $X$ that have $p$-compatible lifts. Let $T$ be the connected component of the center of $G$. That is, $T$ is a torus $\left(\mathbb{C}^{*}\right)^{n}$ and $X \simeq T /(T \cap Y)$ is also a torus $\left(\mathbb{C}^{*}\right)^{n}$ since the group $T \cap Y$ is finite. Let us start with the case when $T \cap Y$ is trivial, i.e. $X=T$. Then $W=X \times Y$ and $p$ is the projection to the first factor. In particular, any "vertical" field $\delta^{\prime} \in \operatorname{AVF}(W, p)$ contains $\mathbb{C}[X] \subset \mathbb{C}[W]$ in its kernel. Every vector field $\sigma \in \operatorname{Lie}_{\omega_{X}}(X)$ has similarly a lift $\sigma^{\prime} \in \operatorname{Lie}_{\omega}(W)$ such that this lift contains $\mathbb{C}[Y]$ in its kernel. In particular, Span Ker $\sigma^{\prime} \cdot \operatorname{Ker} \delta^{\prime}=\mathbb{C}[W]$. Furthermore, any vertical field $\delta^{\prime}$ is of form $\sum_{j} f_{j} \delta_{j}$ where $f_{j} \in \mathbb{C}[W]$ and $\delta_{j}$ is the natural lift of vector field on $Y$ to $W$. Since $\left[\sigma^{\prime}, \delta_{j}\right]=0$ we have $\left[\sigma^{\prime}, \delta^{\prime}\right] \in \operatorname{AVF}(W, p)$ which shows that any rich family of vector fields on $X$ has the desired $p$-compatible lifts.

In the general case when $T \cap Y$ is not trivial we have a commutative diagram

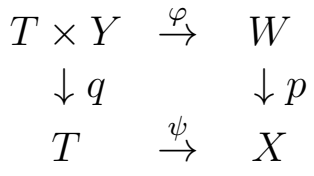

where the vertical arrows are unramified finite coverings. Let $z_{1}, \ldots, z_{n}$ be natural coordinates on $T \simeq\left(\mathbb{C}^{*}\right)^{n}$ and $w_{1}, \ldots, w_{n}$ be natural coordinates on $X \simeq\left(\mathbb{C}^{*}\right)^{n}$. Then up to constant factors we have $w_{j}=\prod_{i=1}^{n} z^{k_{i j}}$. By Example 5.10 a rich family on $X$ consists of vector fields of form $\nu=f_{j} w_{j} \partial / \partial w_{j}$ where $f_{j}$ is a function on $X$ independent of $w_{j}$. Note that $w_{j} \partial / \partial w_{j}=\sum_{i=1}^{n} k_{i j} z_{i} \partial / \partial z_{i}$. Since $z_{i} \partial / \partial z_{i}$ is associated with multiplication by elements of a $\mathbb{C}^{*}$-subgroup of $T$ it may be viewed as a field on $X$ and we can find its lift to $W$. Thus the fields $w_{j} \partial / \partial w_{j}$ and also $\nu$ have lifts to $W$ and we need to check that they are $p$-compatible.

Let $\sigma^{\prime}$ be the lift of one of these fields and $\sigma^{\prime \prime}$ be its preimage on $T \times Y$. Each vertical vector field $\delta^{\prime}$ on $W$ (i.e. it is from the kernel of $p_{*}$ ) generates a vertical vector field $\delta^{\prime \prime}$ on $T \times Y$ (i.e. it is from the kernel of $\left.q_{*}\right)$. As we showed before $\left[\sigma^{\prime \prime}, \delta^{\prime \prime}\right] \in \operatorname{AVF}(T \times Y, q)$ and therefore $\left[\sigma^{\prime}, \delta^{\prime}\right] \in \operatorname{AVF}(W, p)$. Furthermore, since Span $\operatorname{Ker} \sigma^{\prime \prime} \cdot \operatorname{Ker} \delta^{\prime \prime}=\mathbb{C}[T \times Y]$ we have still the equality $\operatorname{Span} \operatorname{Ker} \sigma^{\prime} \cdot \operatorname{Ker} \delta^{\prime}=\mathbb{C}[W]$ by virtue of Lemma 9.4. This concludes the proof of $p$-compatibility and the Lemma.

\section{Compatibility}

7.1. Notation. Let $G$ be a semi-simple Lie group, $S_{0}$ and $S$ be its $S L_{2}$ or $P S L_{2^{-}}$ subgroups, and $p: G \rightarrow X:=G / S_{0}$ be the natural projection into the set of left cosets. Suppose that $\delta$ is a completely integrable algebraic vector field on $S_{0}$ generated by right multiplications. Then it generates $\delta^{\prime} \in \operatorname{Lie}_{\text {alg }}^{\omega}(G, p)$. Let $H \simeq \mathbb{C}_{+}$be a subgroup of $S$. Left multiplication by elements of $H$ generate a locally nilpotent derivation $\sigma^{\prime}$ 
on $G$. Note that $\left[\sigma^{\prime}, \delta^{\prime}\right]=0$ (i.e. we have an $\left(S \times S_{0}\right)$-action on $G$ ) and $\sigma^{\prime}$ generates a locally nilpotent derivation $\sigma$ on $X$ associated with the corresponding $H$-action on $X$.

We think of $S_{0}$ being fixed and our aim is to find "many" $S$ such that $\sigma^{\prime}$ is $p$ compatible for $S$, i.e. the vector space generated by $\operatorname{Ker} \sigma^{\prime} \cdot \operatorname{Ker} \delta^{\prime}$ coincides with $\mathbb{C}[G]$. From now on we use the (seemingly overloaded) notation of strictly semi-compatibility for pairs of vector fields (for Definition see the Appendix) since it was introduced in the work of Donzelli, DvORSKY and the first author [5] and we like to stick to this earlier introduced notation. We apologize for any inconvenience to the reader.

7.2. Lemma. Suppose $g_{0} \in G$ and $S \cap g_{0} S_{0} g_{0}^{-1}=\Gamma$. Then the isotropy group of the point $g_{0} S_{0} \in X$ under the $S$-action is $\Gamma$. In particular, if the $S$-orbit of $g_{0} S_{0}$ is closed then $\Gamma$ is reductive by the Matsushima theorem.

Proof. The coset $g_{0} S_{0}$ is fixed under the action of $s \in S$ if and only if $s g_{0} S_{0} \subset g_{0} S_{0}$ which implies that $g_{0}^{-1} s g_{0} \in S_{0}$ and we have the desired conclusion.

7.3. Proposition. Let $\Gamma_{g}=S \cap g S_{0} g^{-1}$ be finite for every $g \in G$. Then $\sigma^{\prime}$ is $p$ compatible.

Proof. Consider the quotient morphism $r: G \rightarrow Z:=G / /\left(S \times S_{0}\right)$. Since $\Gamma_{g}$ is always finite all orbits are equidimensional and, therefore, closed (indeed, for a reductive group $S \times S_{0}$ the closure of a non-closed orbit must contain a closed orbit, automatically of smaller dimension, which is impossible because all orbits are of the same dimension). By Luna's slice theorem for every point $z \in Z$ there exists a Zariski neigborhood $U \subset Z$, a $\Gamma_{g^{-}}$-invariant slice $V \subset G$ through a point of $r^{-1}(z)$ such that $\left.r\right|_{V}: V \rightarrow U$ is a surjective quasi-finite morphism, and a surjective étale morphism $W \rightarrow r^{-1}(U)$ where $W=V \times_{\Gamma_{g}}\left(S \times S_{0}\right)$. In particular, we have a natural surjective quasi-finite morphism $W^{\prime \prime}:=V \times\left(S \times S_{0}\right) \rightarrow r^{-1}(U)$. Clearly, the algebraic vector fields $\sigma^{\prime \prime}$ and $\delta^{\prime \prime}$ on $W^{\prime \prime}$ induced by $\sigma^{\prime}$ and $\delta^{\prime}$ are strictly semi-compatible, i.e. the span of $\operatorname{Ker} \sigma^{\prime \prime} \cdot \operatorname{Ker} \delta^{\prime \prime}$ coincides with $\mathbb{C}\left[W^{\prime \prime}\right]$. Note also that for any $\mathbb{C}_{+} \simeq H<S$ the quotient $G / / H$ is smooth and the quotient morphism $G \rightarrow G / / H$ is a holomorphic $\mathbb{C}$-fibration over its image. By Lemmas 9.4 and 9.5 in Appendix the restrictions of $\sigma^{\prime}$ and $\delta^{\prime}$ to $r^{-1}(U)$ are also strictly semi-compatible. Thus there is a cover $Z=\bigcup U_{i}$ such that each $U_{i}$ is of form $U_{i}=Z \backslash g_{i}^{-1}(0)$ with $g_{i} \in \mathbb{C}[Z]$ and the restrictions of of $\sigma^{\prime}$ and $\delta^{\prime}$ are strictly semi-compatible on each $W_{i}=r^{-1}\left(U_{i}\right)$. For any function $h \in \mathbb{C}[G]$ its restriction $\left.h\right|_{W_{i}}$ is contained in $\left.\left.\operatorname{Ker} \sigma^{\prime}\right|_{W_{i}} \cap \operatorname{Ker} \delta^{\prime}\right|_{W_{i}}$. Since for any function $\varphi \in \mathbb{C}\left[W_{i}\right]$ there exists $m>0$ such that $\varphi g_{i}^{m} \in \mathbb{C}[G]$ and since $g_{i} \in \operatorname{Ker} \sigma^{\prime} \cap \operatorname{Ker} \delta^{\prime}$, for an appropriate $m$ the function $h g_{i}^{m}$ belongs to the span of $\operatorname{Ker} \sigma^{\prime} \cdot \operatorname{Ker} \delta^{\prime}$. Now the desired conclusion follows from the standard application of the Nullstellensatz.

7.4. Remark. Lemmas 9.4 and 9.5 are to a great extend repetitions of Lemmas 3.6 and Lemma 3.7 in [12]. Therefore, we put them in Appendix. 
7.5. Lemma. Let $G, S_{0}, X$, and $S$ be as in Lemma 7.2 and $\Gamma_{g}=S \cap g S_{0} g^{-1}$ where $g \in G$. Suppose that $\Gamma_{g}$ does not contain a torus $\mathbb{C}^{*}$ for every $g \in G$. Then every $\Gamma_{g}$ is finite.

Proof. Assume that $\Gamma_{g_{0}}$ is not finite for some $g_{0} \in G$. Then $\Gamma_{g_{0}}$ cannot be reductive (without a torus) and the $S$-orbit $O$ of $g_{0} S_{0} \in X$ is not closed by the second statement of Lemma 7.2. Furthermore, since any two-dimensional subgroup of $S L_{2}(\mathbb{C})$ contains $\mathbb{C}^{*}$ we see that $\Gamma_{g_{0}}$ is one-dimensional, i.e. $O$ is two-dimensional. Since $S$ is reductive the closure of $O$ must contain a closed orbit $O_{1}$ of some point $g_{1} S_{0} \in X$. Thus $\operatorname{dim} O_{1} \leq 1$ and $\operatorname{dim} \Gamma_{g_{1}} \geq 2$. But in this case as we mentioned $\Gamma_{g_{1}}$ contains a torus which yields a contradiction.

In order to find $S$ such that $\Gamma_{g}=g^{-1} S_{0} g \cap S$ does not contain a torus for every $g \in G$ we need to remind the notion of a principal $S L_{2}$ or $P S L_{2}$-subgroup of a semi-simple group $G$ (resp. principal $\mathfrak{s l}_{2}$-subalgebra in the Lie algebra $\mathfrak{g}$ of $G$ ) from [4. Recall that a semi-simple element $h$ of $\mathfrak{g}$ is called regular if the dimension of its centralizer is equal to the rank of $\mathfrak{g}$ (more precisely, this centralizer coincides with a Cartan subalgebra $\mathfrak{h}$ of $\mathfrak{g})$. An $\mathfrak{s l}_{2}$ subalgebra $\mathfrak{s}$ of $\mathfrak{g}$ is called principal if it contains a regular semi-simple element $h$ such that every eigenvalue of its adjoint operator is an even integer. The $S L_{2}$ (or $P S L_{2}$ ) subgroup generated by such subalgebra is also called principal. For instance, in $S L_{n}$ up to conjugation every regular element is a diagonal matrix with distinct eigenvalues and any principal $S L_{2}$-subgroup acts irreducibly on the natural $n$-space. Any two principal $S L_{2}$-subgroups are conjugated in $G$ and any $S L_{2}$-subgroup corresponding to a root is not principal (unless $\mathfrak{g}=\mathfrak{s l}_{2}$ ) since its semi-simple elements are not regular.

7.6. Lemma. If $S$ is a principal $S L_{2}$ (resp. $P S L_{2}$ ) subgroup of a semi-simple group $G$ and $S_{0}$ be any subgroup of $G$ that does not contain regular semi-simple elements. Then $\Gamma_{g}=g^{-1} S_{0} g \cap S$ is finite for every $g \in G$.

Proof. Note that $\Gamma_{g}$ cannot contain a torus since otherwise $S_{0}$ contains a regular semisimple element. Lemma 7.5 implies now the desired conclusion.

7.7. Proposition. Let $G$ be a semi-simple Lie group different from $S L_{2}(\mathbb{C})$ or $P S L_{2}(\mathbb{C})$. Suppose that $S_{0}, Z=G / S_{0}, p: G \rightarrow Z$, and $\sigma^{\prime}$ are is in Notation 7.1. Let $S_{0}$ correspond to a root in the Dynkin diagram. Then $\sigma^{\prime}$ can be chosen that it is p-compatible (for any $S_{0}$ corresponding to a root in the Dynkin diagram!!). Furthermore, there are enough of these $p$-compatible completely integrable algebraic vector fields $\sigma^{\prime}$, so that the Lie algebra $L$ generated by them generates $\mathrm{VF}_{\mathrm{alg}}(Z)$ as a $\mathbb{C}[Z]$-module.

Proof. Let an $S L_{2}$ (or $P S L_{2}$ ) subgroup $S_{0}$ correspond to a root and $S$ be a principal $S L_{2}$ (or $P S L_{2}$ ) subgroup. By Proposition 7.3 and Lemma [7.6 $\sigma^{\prime}$ is $p$-compatible and we are left with the second statement. Suppose that $X, Y, H$ is a standard triple in the 
$\mathfrak{s l}_{2}$-Subalgebra $\mathfrak{s}$ of $S$, i.e. $[X, Y]=H,[H, X]=2 X,[H, Y]=-2 Y$ 6. In particular, the locally nilpotent vector fields generated by $X$ and $Y$ are of form $\sigma^{\prime}$ and they are $p$-compatible. Suppose that the centralizer of $H$ is the Cartan subalgebra $\mathfrak{h}$ associated with the choice of root system and $X_{0}, Y_{0}, H_{0}$ is an $\mathfrak{s l}_{2}$-triple corresponding to one of the roots. Conjugate $S$ by $x_{0}=e^{\varepsilon X_{0}}$ where $\varepsilon$ is a small parameter. Up to terms of order 2 element $H$ goes to $H+\varepsilon\left[H, X_{0}\right]$ after such conjugation, i.e. $\left[H, X_{0}\right]$ belongs (up to second order) to the Lie algebra generated by $X, Y$, and the nilpotent elements of the Lie algebra of principal $S L_{2}$-subgroup $x_{0}^{-1} S x_{0}$. Since each $X_{0}$ is an eigenvector of the adjoint action of $H$ we have $\left[H, X_{0}\right]=a X_{0}$. Furthermore, $a \neq 0$ since otherwise $X_{0}$ belongs to the centralizer $\mathfrak{h}$ of the regular element $H$. Thus $X_{0}$ and similarly $Y_{0}$ are (up o second order) in the Lie algebra $L$ generated by fields of form $\sigma^{\prime}$. The same is true for $H_{0}=\left[X_{0}, Y_{0}\right]$. Thus values of $L$ at any point $z \in Z$ generate the tangent space $T_{z} Z$ which implies $L$ generates $\operatorname{VF}_{\text {alg }}(Z)$ as a $\mathbb{C}[Z]$-module.

\section{Main Theorem}

8.1. Notation. In this section $G$ is a semi-simple Lie group. By $S_{i}$ we denote an $S L_{2}$ or $P S L_{2}$-subgroup of $G$ (for each index $i \geq 0$ ) and by $p_{i}: G \rightarrow X_{i}=G / S_{i}$ the natural projection. By abusing notation we treat $\mathbb{C}\left[X_{i}\right]$ as the subring $p_{i}^{*}\left(\mathbb{C}\left[X_{i}\right]\right)$ in $\mathbb{C}[G]$. Note that Lemma 5.12 implies that $\mathbb{C}[G] \simeq F\left(G, p_{i}\right) \oplus \mathbb{C}\left[X_{i}\right]$ and denote by $\mathrm{pr}_{i}: \mathbb{C}[G] \rightarrow \mathbb{C}\left[X_{i}\right]$ the natural projection. For any semi-simple complex Lie group $B$ denote by $B^{\mathbb{R}}$ its maximal compact subgroup whose complexification coincides with $B$ (it is unique up to conjugation). Let $K_{i}=S_{i}^{\mathbb{R}}$. Define a linear operator $\mathrm{av}_{i}: \mathbb{C}[G] \rightarrow \mathbb{C}[G]$ by

$$
\operatorname{av}_{i}(f)=\int_{K_{i}} f(w k) \mathrm{d} \mu_{K_{i}}(k)
$$

for any function $f \in \mathbb{C}[G]$ where $\mu_{K_{i}}(k)$ is the bi-invariant normalized Haar measure on $K_{i}$.

8.2. Lemma. In Notation 8.1 we have

(i) the right multiplication by an element $k \in K_{i}$ generates a map $\Psi: \mathbb{C}[G] \rightarrow \mathbb{C}[G]$ (given by $f(w) \rightarrow f(w k)$ ) whose restriction to $F\left(G, p_{i}\right)$ is an isomorphism;

(ii) Ker $\operatorname{av}_{i}=F\left(G, p_{i}\right)$, i.e. $\operatorname{av}_{i}=\operatorname{pr}_{i}$ and $f-\operatorname{av}_{i}(f) \in F\left(G, p_{i}\right)$ for every $f \in \mathbb{C}[G]$.

Proof. The right multiplication transforms every fiber $Y:=p_{i}^{-1}(x)$ into itself and each completely integrable algebraic vector field on it into a similar field. Hence for every $f \in F\left(G, p_{i}\right)$ we have $\left.\Psi(f)\right|_{p_{i}^{-1}(x)} \in F_{Y}$. Now (i) follows from Lemma 5.12, Thus operator $\mathrm{av}_{i}$ respects the direct sum $\mathbb{C}[G] \simeq F\left(G, p_{i}\right) \oplus \mathbb{C}\left[X_{i}\right]$ and sends $\mathbb{C}[G]$ onto $\mathbb{C}\left[X_{i}\right]$ so that its restriction to $\mathbb{C}\left[X_{i}\right]$ is identical map. This implies (ii).

\footnotetext{
${ }^{6}$ It is unfortunate, but we have to use the classical notation $X, Y, H$ for a standard triple of an $\mathfrak{s l}_{2}$-algebra while in the rest of the text these symbols denote affine algebraic varieties and groups.
} 
8.3. Lemma. Let $S_{0}$ and $K_{0}$ be as before and let $L=G^{\mathbb{R}}$ contain $K_{0}$. Consider the natural inner product on $\mathbb{C}[G]$ given by

$$
h_{1} \cdot h_{2}=\int_{l \in L} h_{1}(l) \bar{h}_{2}(l) \mathrm{d} \mu_{L}(l)
$$

where $\mu_{L}$ is the bi-invariant measure on $L$. Then $\mathbb{C}\left[X_{0}\right]$ is the orthogonal complement of $F\left(G, p_{0}\right)$.

Proof. Consider $\left.h_{1} \in \mathbb{C}[G]\right)$ and $h_{2} \in \mathbb{C}\left[X_{0}\right]$. Show that $\operatorname{av}_{0}\left(h_{1}\right) \cdot h_{2}=h_{1} \cdot h_{2}$. We have

$$
I:=\operatorname{av}_{0}\left(h_{1}\right) \cdot h_{2}=\int_{L} \int_{K_{0}} h_{1}\left(l k_{0}\right) \bar{h}_{2}(l) \mathrm{d} \mu_{K_{0}}\left(k_{0}\right) \mathrm{d} \mu_{L}(l) .
$$

By Fubini's theorem

$$
I=\int_{K_{0}} \int_{L} h_{1}\left(l k_{0}\right) \bar{h}_{2}(l) \mathrm{d} \mu_{L}(l) \mathrm{d} \mu_{K_{0}}\left(k_{0}\right) .
$$

Set $l^{\prime}=l k_{0}$. Then $h_{1}\left(l k_{0}\right)=h_{1}\left(l^{\prime}\right)$ and $h_{2}(l)=h_{2}\left(l^{\prime} k_{0}^{-1}\right)=h_{2}\left(l^{\prime}\right)$ since $h_{2}$ is right $K_{0}$-invariant. Using the fact that measures are invariant we see that $I$ coincides with

$$
\begin{gathered}
\int_{K_{0}} \int_{L} h_{1}\left(l^{\prime}\right) \bar{h}_{2}\left(l^{\prime}\right) \mathrm{d} \mu_{L}\left(l^{\prime}\right) \mathrm{d} \mu_{K_{0}}\left(k_{0}\right)= \\
\left.\int_{L} h_{1}\left(l^{\prime}\right) \bar{h}_{2}\left(l^{\prime}\right)\right) \mathrm{d} \mu_{L}\left(l^{\prime}\right)
\end{gathered}
$$

where the last equality holds since measure $\mu_{K_{0}}$ is normalized. Thus av $\left(h_{1}\right) \cdot h_{2}=$ $h_{1} \cdot h_{2}$. Now the desired conclusion follows from Lemma 8.2 and the fact that $\mathbb{C}[G] \simeq$ $F\left(G, p_{0}\right) \oplus \mathbb{C}\left[X_{0}\right]$.

8.4. Corollary. Let $S_{0}, \ldots, S_{m}$ be as in Notation 8.1 with each $K_{i}=S_{i}^{\mathbb{R}} \subset$ L. Set $F=\sum_{i=0}^{m} F\left(G, p_{i}\right)$. Then the orthogonal complement of $F$ in $\mathbb{C}[G]$ coincides with the subspace $V$ of functions that are invariant with respect to any $S_{i}$-action generated by the right multiplication. In particular, if this set $S_{0}, \ldots, S_{m}$ contains all $S L_{2}$ or $P S L_{2}$ subgroups corresponding to positive (or even simple positive) roots for the Lie algebra of $G$ then this orthogonal complement $V$ consists of constants only and $\mathbb{C}[G] \simeq F \oplus \mathbb{C}$.

Proof. Indeed, treating $\mathbb{C}\left[X_{i}\right]$ as $p_{i}^{*}\left(\mathbb{C}\left[X_{i}\right]\right)$ we see that by Lemma 8.3 the orthogonal complement of $F$ in $\mathbb{C}[G]$ is $V=\bigcap_{i=0}^{m} \mathbb{C}\left[X_{i}\right]$ which is exactly the space of functions invariant under each $S_{i}$-action. For the second statement note that if the sequence $\left\{S_{i}\right\}$ of subgroups generate the whole group $G$ then these invariant functions must be constants.

8.5. Lemma. Let $\left\{S_{i}\right\}_{i=0}^{m}, F$, and $V$ be as in Corollary 8.4 and $f_{0} \in \mathbb{C}[G] \backslash F$. Consider the smallest subspace $U \subset \mathbb{C}[G]$ that contains $f_{0}$ and such that for every $i$ and every $f \in U$ function $\operatorname{av}_{i}(f)$ is also in $U$. Then

(1) $U$ is of some finite dimension $N$;

(2) $\operatorname{dim} U \cap F=N-1$ and $\operatorname{dim} U \cap V=1$. 
Proof. Consider a closed embedding $\rho: G \hookrightarrow \mathbb{C}^{n}$ such that the induced action of $G$ on $\mathbb{C}^{n}$ is linear. This yields a filtration on $\mathbb{C}[G]$ defined by minimal degrees of polynomial extensions of regular functions on $G$ to $\mathbb{C}^{n}$. Let $W_{k}$ be the subspace of $\mathbb{C}[G]$ that consists of functions of degree at most $k$ and $\Phi_{l}: \mathbb{C}[G] \rightarrow \mathbb{C}[G]$ be the automorphism given by $f(w) \rightarrow f(w l)$ for $l \in L$. Since the $G$-action on $\mathbb{C}^{n}$ is linear each automorphism $\Phi_{l}$ sends $W_{k}$ into itself. Hence the definition of $\operatorname{av}_{i}$ implies that $\operatorname{av}_{i}\left(W_{k}\right) \subset W_{k}$. Thus $U \subset W_{k}$ as soon as $f_{0} \in W_{k}$ which yields (1).

Denote the orthogonal projection onto $V$ by pr: $\mathbb{C}[G] \rightarrow V$ and let $f_{0}^{\prime}=\operatorname{pr}\left(f_{0}\right)$. Since $f_{0} \notin F$ we have $f_{0}^{\prime} \neq 0$. Let $P$ be the hyperplane in $\mathbb{C}[G]$ that consists of vectors of form $f_{0}^{\prime}+P_{0}$ where $P_{0}$ is the hyperspace orthogonal to $f_{0}^{\prime}$. In particular $P$ contains $f_{0}$. Since $f_{0}^{\prime} \in \mathbb{C}\left[X_{i}\right]$ for every $i$ we see that $P$ is orthogonal to each $\mathbb{C}\left[X_{i}\right]$. Recall that the operator $\mathrm{av}_{i}=\mathrm{pr}_{i}$ is just the orthogonal projection to $\mathbb{C}\left[X_{i}\right]$, i.e. $P$ is invariant with respect to these operators. In particular, if we set $f_{J}=\operatorname{av}_{j_{1}} \circ \cdots \circ \operatorname{av}_{j_{s}}\left(f_{0}\right)$ for a multi-index $J=\left(j_{1}, \ldots, j_{s}\right)$ with $j_{t} \in\{0, \ldots, m\}$ then $f_{J} \in P \cap U$.

We want to show that for some sequence of such multi-indices $f_{J}$ is convergent to a nonzero element of $V$ or, equivalently, $f_{J}^{\prime}$ is convergent to an element of $V \cap P_{0}$ for $f_{J}^{\prime}=f_{J}-f_{0}^{\prime}$. Consider the subspace $U^{\prime}$ generated by vectors of form $f_{J}^{\prime}$. Let $U_{i}^{\prime}=U^{\prime} \cap \mathbb{C}\left[X_{i}\right]$ and $I=(J, i)$, i.e. $f_{I}^{\prime}=\operatorname{av}_{i}\left(f_{J}^{\prime}\right)$. By construction the operator $\left.\operatorname{pr}_{i}\right|_{U^{\prime}}=\left.\operatorname{av}_{i}\right|_{U^{\prime}}$ is just the orthogonal projection to $U_{i}^{\prime}$. Hence if $f_{J}^{\prime} \notin U_{i}^{\prime}$ we have $\left\|f_{I}^{\prime}\right\|<\left\|f_{J}^{\prime}\right\|$. Since $U^{\prime}$ is finite-dimensional this implies that one can choose $\left\{f_{J}\right\}$ convergent to an element $v \in U^{\prime}$ and we can suppose that $v$ has the smallest possible norm. Then $\operatorname{pr}(v)=v$ because of the last inequality. On the other hand $\operatorname{pr}\left(f_{J}^{\prime}\right)=$ $\operatorname{pr}\left(f_{J}\right)-\operatorname{pr}\left(f_{0}^{\prime}\right)=\operatorname{pr} \circ \operatorname{av}_{j_{1}} \circ \cdots \circ \operatorname{av}_{j_{s}} \circ\left(f_{0}\right)-\operatorname{pr}\left(f_{0}\right)=f_{0}^{\prime}-f_{0}^{\prime}=0$. Thus $v=0$. This shows $f_{0}^{\prime} \in V \cap U$ and therefore $\operatorname{dim} U \cap V \geq 1$.

On the other hand $U$ contains a subspace $U_{0}$ generated by vectors of form $f_{0}-f_{J}$. One can see that $U_{0}$ is of codimension 1 in $U$. Furthermore, $f_{0}-f_{I}=\left(f_{0}-f_{J}\right)+\left(f_{J}-f_{I}\right)$. Note that $f_{J}-f_{I}=f_{J}-\operatorname{av}_{i}\left(f_{J}\right) \in F\left(G, p_{i}\right) \subset F$ by Lemma 8.2. Thus, using induction by the length of the multi-index $J$ one can show that $f_{0}-f_{J} \in F$. That is, $\operatorname{dim} U \cap F \geq$ $N-1$ which concludes the proof.

8.6. Proposition. Any semi-simple group $G$ has the algebraic volume density with respect to the invariant volume.

Proof. Choose $S_{0}, \ldots, S_{m}$ as in Corollary 8.4 and such that they correspond to simple nodes in the Dynkin diagram (it is possible since every semi-simple group $G$ has a compact real form, i.e. we can suppose that $S_{i}^{\mathbb{R}}=K_{i} \subset L=G^{\mathbb{R}}$ ). Consider the natural projections $p_{i}: G \rightarrow X_{i}:=G / S_{i}$ to the sets of left cosets. Choose $p_{i}$-compatible completely integrable algebraic vector fields $\sigma^{\prime}$ as in Proposition 7.7 and denote their collection by $\Theta$. That is, vector fields from $\Theta$ are of zero divergence, they commute with any $\delta \in \operatorname{VF}_{\text {alg }}^{\omega}\left(G, p_{i}\right)$, and they are independent from index $i$. Furthermore, these fields from $\Theta$ can be viewed also as zero divergence vector fields on $X_{i}$ that generate $\mathrm{VF}_{\text {alg }}\left(X_{i}\right)$ as a $\mathbb{C}\left[X_{i}\right]$-module. 
Let us fix an index $i$. Any algebraic vector field on $G$ is of form

$$
\nu=\sum_{\theta \in \Theta} h_{\theta} \theta+\delta
$$

where the sum contains only finite number of nonzero terms, $h_{\theta} \in \mathbb{C}[G]$ and $\delta \in$ $\mathrm{VF}_{\text {alg }}\left(G, p_{i}\right)$. Since $S L_{2}(\mathbb{C})$ and $P S L_{2}(\mathbb{C})$ are fine, $\mathbb{C}[G]=F\left(G, p_{i}\right) \oplus \mathbb{C}\left[X_{i}\right]$ by Lemma 5.12. Thus by virtue of Proposition 5.16 adding fields from $\operatorname{Lie}_{a l g}^{\omega}(G)$ to $\nu$ we get a field

$$
\nu_{i}=\sum_{\theta \in \Theta} h_{\theta}^{i} \theta+\delta_{i}
$$

where $\delta_{i} \in \operatorname{VF}_{\mathrm{alg}}^{\omega}\left(G, p_{i}\right), h_{\theta}^{i}=\mathrm{av}_{i}\left(h_{\theta}\right)$, and $\mathrm{av}_{i}=\mathrm{pr}_{i}$ is as Notation 8.1. That is, $h_{\theta}^{i} \in \mathbb{C}\left[X_{i}\right]$. Suppose that $\operatorname{div}_{\omega} \nu=0$ and, therefore, $\operatorname{div}_{\omega}\left(\nu_{i}\right)=0$. Note that

$$
\operatorname{div}_{\omega}\left(\sum_{\theta \in \Theta} h_{\theta}^{i} \theta\right)=\sum_{\theta \in \Theta} \theta\left(h_{\theta}^{i}\right) \in \mathbb{C}\left[X_{i}\right]
$$

while $\operatorname{div}_{\omega} \delta_{i} \in F\left(G, p_{i}\right)$. Hence $\operatorname{div}_{\omega}\left(\sum_{\theta \in \Theta} h_{\theta}^{i} \theta\right)=\operatorname{div}_{\omega}\left(\delta_{i}\right)=0$. Since $S L_{2}(\mathbb{C})$ and $P S L_{2}(\mathbb{C})$ have the algebraic volume density property, $\delta_{i} \in \operatorname{Lie}^{\omega}(G, p)$ by Corollary 5.14. Thus $\nu-\tilde{\nu}_{i} \in \operatorname{Lie}_{\text {alg }}^{\omega}(G)$ where

$$
\tilde{\nu}_{i}=\sum_{\theta \in \Theta} h_{\theta}^{i} \theta
$$

In particular it suffices to show that $\tilde{\nu}_{i} \in \operatorname{Lie}_{\text {alg }}^{\omega}(G)$ and, therefore, we can suppose that $\delta=0$ in the original formula for $\nu$. Repeating this procedure we see that $\nu-\tilde{\nu}_{J} \in$ $\operatorname{Lie}_{\text {alg }}^{\omega}(G)$ where

$$
\tilde{\nu}_{J}=\sum_{\theta \in \Theta} h_{\theta}^{J} \theta
$$

for a multi-index $J=\left(j_{1}, \ldots, j_{s}\right)$ with $j_{t} \in\{0, \ldots, m\}$ and $h_{\theta}^{J}=\operatorname{av}_{j_{1}} \circ \cdots \circ \operatorname{av}_{j_{s}}\left(h_{\theta}\right)$.

By Corollary 8.4 and Lemma 8.5 the vector space generated by $h_{\theta}$ and functions of form $h_{\theta}^{J}$ is also generated by constants and functions of form $h_{\theta}-h_{\theta}^{J}$. Thus adding to $\nu=\sum_{\theta \in \Theta} h_{\theta} \theta$ vector fields of form $\nu-\tilde{\nu}_{\theta}^{J}$ and $c \theta$ (where $c \in \mathbb{C}$ ) we can reduce the number of nonzero terms in this sum. Hence $\nu \in \operatorname{Lie}_{a l g}^{\omega}(G)$ which implies the desired conclusion.

8.7. Proof of Theorem 2. Let us start with the case when $G$ is reductive. Suppose that $Y$ is its Levi semi-simple subgroup. Then by Proposition $6.11 p: G \rightarrow X:=G / Y$ is a refined volume fibration and by Theorem $4 G$ has the algebraic volume density property.

Now consider an arbitrary linear algebraic group $G$ and let $Y$ be its unipotent ideal and $X$ be a Levi reductive subgroup of $G$. By Corollary 6.8 the Mostow isomorphism $G \rightarrow X \times Y$ makes the left invariant volume $\omega$ on $G$ equal to $\omega_{X} \times \omega_{Y}$ where $\omega_{X}$ is left invariant on $X$ and $\omega_{Y}$ is invariant on $Y$. Now by Proposition $4.4 G$ has the algebraic volume density property with respect to $\omega$ which concludes the proof of our Main Theorem. 
8.8. Remark. Theorem 2 remains, of course, valid if instead of the left invariant volume form we consider the right invariant one, because the affine automorphism $G \rightarrow G, g \rightarrow g^{-1}$ transforms the left invariant volume form into the right one while preserving the complete integrability of the algebraic vector fields.

\section{Appendix: Strictly SEMi-COMPatible FieldS}

9.1. Notation. In this section $H_{i}$ is isomorphic to $\mathbb{C}_{+}$for $i=1,2$. We suppose also that $X$ is a normal affine algebraic variety equipped with nontrivial algebraic $H_{i}$-actions (in particular, each $H_{i}$ generates an algebraic vector field $\delta_{i}$ on $X$ ). The categorical quotients will be denoted $X_{i}=X / / H_{i}$ and the quotient morphisms by $\rho_{i}: X \rightarrow X_{i}$.

9.2. Definition. A pair $\left(\delta_{1}, \delta_{2}\right)$ of algebraic vector fields (as in Notation 9.1) is called strictly semi-compatible if the span of $\operatorname{Ker} \delta_{1} \cdot \operatorname{Ker} \delta_{2}$ coincides with $\mathbb{C}[X]$.

We shall need the following obvious geometric reformulation of Definition.

9.3. Proposition. Let $\delta_{1}$ and $\delta_{2}$ be as in Notation 9.1. Set $\rho=\left(\rho_{1}, \rho_{2}\right): X \rightarrow Y:=$ $X_{1} \times X_{2}$ and $Z$ equal to the closure of $\rho(X)$ in $Y$. Then $\delta_{1}$ and $\delta_{2}$ are strictly semicompatible if and only if $\rho: X \rightarrow Z$ is an isomorphism.

9.4. Lemma. Let $X, H_{i}, X_{i}, \delta_{i}$, and $\rho_{i}$ be as in Notation 9.1 with $\delta_{1}$ and $\delta_{2}$ being strictly semi-compatible and $\left[\delta_{1}, \delta_{2}\right]=0$. Set $\Gamma=H_{1} \times H_{2}$. Let $X^{\prime}$ be a normal affine algebraic variety equipped with a non-degenerate $\Gamma$-action and $p: X \rightarrow X^{\prime}$ be a finite $\Gamma$-equivariant morphism (for each $i=1,2$ ), i.e. we have commutative diagrams

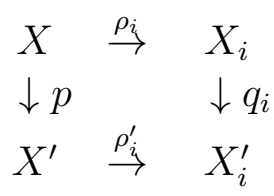

where $\rho_{i}^{\prime}: X^{\prime} \rightarrow X_{i}^{\prime}=X^{\prime} / / H_{i}$ is the quotient morphism of the $H_{i}$-action on $X^{\prime}$. Suppose also that $\rho_{1}^{\prime}$ makes $X^{\prime}$ an étale locally trivial $\mathbb{C}$-fibration over $\rho_{1}^{\prime}\left(X^{\prime}\right)$, and $X_{1}, X_{2}$ are affine $]$. Then $\operatorname{Span}\left(\mathbb{C}\left[X_{1}^{\prime}\right] \cdot \mathbb{C}\left[X_{2}^{\prime}\right]\right)=\mathbb{C}\left[X^{\prime}\right]$.

Proof. Since $p$ is finite, every $f \in \mathbb{C}\left[X_{i}\right] \subset \mathbb{C}[X]$ is a root of a minimal monic polynomial with coefficients in $\mathbb{C}\left[X^{\prime}\right]$ that are constant on $H_{i}$-orbits (since otherwise $f$ is not constant on these orbits). By the universal property these coefficients are regular on $X_{i}^{\prime}$, i.e. $f$ is integral over $\mathbb{C}\left[X_{i}^{\prime}\right]$ and $q_{i}$ is finite. Consider the commutative diagram

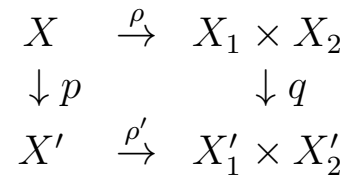

where $\rho=\left(\rho_{1}, \rho_{2}\right), \rho^{\prime}=\left(\rho_{1}^{\prime}, \rho_{2}^{\prime}\right)$, and $q=\left(q_{1}, q_{2}\right)$. Let $Z$ (resp. $Z^{\prime}$ ) be the closure of $\rho(X)$ in $X_{1} \times X_{2}$ (resp. $\rho^{\prime}\left(X^{\prime}\right)$ in $\left.X_{1}^{\prime} \times X_{2}^{\prime}\right)$. By Proposition $0.3 \rho: X \rightarrow Z$ is an

\footnotetext{
${ }^{7}$ In all cases we apply this Lemma the $\mathbb{C}_{+}$-action generated by $H_{i}$ extends to an algebraic $S L_{2}(\mathbb{C})$ action and, therefore, $X_{i}$ is affine automatically by the Hadziev theorem [11.
} 
isomorphism and, therefore, by Lemma 3.6 in [12] $\rho^{\prime}: X^{\prime} \rightarrow Z^{\prime}$ is birational finite. Since the statement of this Lemma is equivalent to the fact that $\rho^{\prime}$ is an isomorphism, it suffices to prove $\rho^{\prime}$ is a holomorphic embedding.

Consider an orbit $O \subset X$ of $H_{1}$ and set $O^{\prime}=p(O), O_{2}^{\prime}=\rho_{2}^{\prime}\left(O^{\prime}\right)$. Each of these orbits is isomorphic to $\mathbb{C}_{+}$and, therefore, the $H_{1}$-equivariant finite morphisms $\left.p\right|_{O}$ : $O \rightarrow O^{\prime}$ and $\left.\rho_{2}^{\prime}\right|_{O^{\prime}}: O^{\prime} \rightarrow O_{2}^{\prime}$ must be isomorphisms. Thus one has a regular function on $X_{2}^{\prime}$ whose restriction yields a coordinate on $O^{\prime} \simeq O_{2}^{\prime} \subset X_{2}^{\prime}$. Since locally $X^{\prime}$ is biholomorphic to $U \times O^{\prime}$ where $U$ is an open subset of $\rho_{1}^{\prime}\left(X^{\prime}\right) \subset X_{1}^{\prime}$ we see that $\rho^{\prime}: X^{\prime} \rightarrow X_{1}^{\prime} \times X_{2}^{\prime}$ is a local holomorphic embedding, i.e. it remains to show that $\rho^{\prime}$ is injective. For any $x \in X$ set $x^{\prime}=p(x)$, and $x_{i}^{\prime}=\rho_{i}^{\prime}\left(x^{\prime}\right)$. Assume that $x$ and $y \in X$ are such that $\left(x_{1}^{\prime}, x_{2}^{\prime}\right)=\left(y_{1}^{\prime}, y_{2}^{\prime}\right)$. Arguing as in Lemma 3.68 in [12] we can suppose that $x$ and $y$ belong to the same fiber of $\rho_{1}$ that is, by assumption, an $H_{1}$-orbit $O$. Since $\left.\rho_{2}^{\prime}\right|_{O^{\prime}}: O^{\prime} \rightarrow O_{2}^{\prime}$ is an isomorphism we have $x^{\prime}=y^{\prime}$ which implies the desired conclusion.

9.5. Lemma. Let the assumption of Lemma 9.4 hold with one exception: instead of the finiteness of $p$ we suppose that there are a surjective quasi-finite morphism $r: S \rightarrow S^{\prime}$ of normal affine algebraic varieties equipped with trivial $\Gamma$-actions and a surjective $\Gamma$ equivariant morphism $\varrho^{\prime}: X^{\prime} \rightarrow S^{\prime}$ such that $X$ is isomorphic to fibred product $X^{\prime} \times_{S^{\prime}} S$ with $p: X \rightarrow X^{\prime}$ being the natural projection (i.e. $p$ is surjective quasi-finite). Then the conclusion of Lemma 9.4 remains valid.

Proof. By construction, $X_{i}=X_{i}^{\prime} \times{ }_{S^{\prime}} S$. Thus we have the following commutative diagram

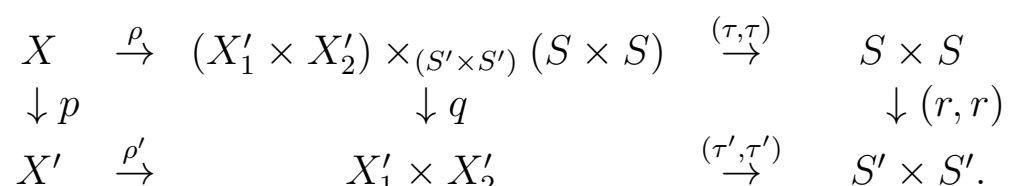

Set $Z$ (resp. $Z^{\prime}$ ) equal to the closure of $\rho(X)$ in $X_{1} \times X_{2}\left(\operatorname{resp} . \rho^{\prime}\left(X^{\prime}\right)\right.$ in $\left.X_{1}^{\prime} \times X_{2}^{\prime}\right)$ and $D \simeq S$ (resp. $\left.D^{\prime} \simeq S^{\prime}\right)$ be the diagonal subset in $S \times S$ (resp. $\left.S^{\prime} \times S^{\prime}\right)$. Since $X=X^{\prime} \times{ }_{S^{\prime}} S$ we see that $Z=Z^{\prime} \times{ }_{D^{\prime}} D$.

Assume that $\rho^{\prime}\left(x^{\prime}\right)=\rho^{\prime}\left(y^{\prime}\right)=: z^{\prime}$ for some $x^{\prime}, y^{\prime} \in X^{\prime}$. Then by the commutativity of the diagram we have also $\varrho^{\prime}\left(x^{\prime}\right)=\varrho^{\prime}\left(y^{\prime}\right)=: s^{\prime}$. Since $r$ is surjective $r(s)=s^{\prime}$ for some $s \in S$. Thus the elements $\left(x^{\prime}, s\right)$ and $\left(y^{\prime}, s\right)$ of $X^{\prime} \times{ }_{S^{\prime}} S$ go to the same element $\left(z^{\prime}, s^{\prime}\right)$ of $Z^{\prime} \times{ }_{D^{\prime}} D$ under morphism $\rho$. By Lemma 9.3 $\rho: X \rightarrow Z$ is an isomorphism and therefore $x^{\prime}=y^{\prime}$. Hence $\rho^{\prime}: X^{\prime} \rightarrow Z^{\prime}$ is bijective 9 . It was shown in the proof of Lemma 9.4 that $\rho^{\prime}$ is locally biholomorphic, i.e. it is an isomorphism which implies the desired conclusion.

\footnotetext{
${ }^{8}$ It is shown in that Lemma that $\rho_{j}\left(p^{-1}\left(x^{\prime}\right)\right)=q_{j}^{-1}\left(x_{j}^{\prime}\right)$ for a general point $x \in X$ and to adjust the argument to the present situation one needs it to be true for every point in $X$, but this follows, of course, from continuity and finiteness of $q_{j}$.

${ }^{9}$ Note that this (slightly modified) argument provides a much simpler proof of Lemma 3.7 in [12] where assuming that $\rho$ is birational finite one needs to show that $\rho^{\prime}$ is such.
} 


\section{REFERENCES}

[1] E. Andersén, Volume-preserving automorphisms of $\mathbb{C}^{n}$, Complex Variables Theory Appl. 14 (1990), no. 1-4, 223-235.

[2] E. Andersén, Complete vector fields on $\left(C^{*}\right)^{n}$, Proc. Amer. Math. Soc. 128 (2000), no. 4, 10791085.

[3] E. Andersén, L. Lempert, On the group of holomorphic automorphisms of $\mathbb{C}^{n}$, Invent. Math. 110 (1992), no. 2, 371-388.

[4] N. Bourbaki, Elements of Mathematics. Lie Groups and Lie Algebras. Chapters 7-9., Springer, Berlin-Heidelberg-New York, 2005.

[5] F. Donzelli, A. Dvorsky, S. Kaliman, Algebraic density property of homogeneous spaces, to appear.

[6] F. Forstnerič A theorem in complex symplectic geometry, J. Geom. Anal. 5 (1995), no. 3, 379-393.

[7] F. Forstnerič, J.-P. Rosay, Approximation of biholomorphic mappings by automorphisms of $\mathbb{C}^{n}$, Invent. Math. 112 (1993), no. 2, 323-349.

[8] W. Fulton, J. Harris, Representation Theory. A First Course, Graduate Texts in Mathematics, 129, Readings in Mathematics, Springer-Verlag, New York, 1991, 551 pp.

[9] A. Grothendieck, On the de Rham cohomology of algebraic varieties, Inst. Hautes Etudes Sci. Publ. Math., 29 (1966), 95-105.

[10] R. Hartshorne, Algebraic Geometry, Springer-Verlag, New York Berlin Heidelberg London, 1977, 496p.

[11] Dž. Hadžiev, Certain questions of the theory of vector invariants, (Russian) Mat. Sbornik, 72 (1967) 420-435.

[12] S. Kaliman, F. Kutzschebauch Criteria for the density property of complex manifolds, Invent. Math. 172 (2008), no. 1, 71-87.

[13] S. Kaliman, F. Kutzschebauch Density property for hypersurfaces uv $=p(\bar{x})$, Math. Z. 258 (2008), no. $1,115-131$.

[14] S. Kaliman, M. Zaidenberg, Affine modifications and affine hypersurfaces with a very transitive automorphism group, Trans. Groups 4 (1999), 53-95.

[15] Sh. Kobayashi, K. Nomizu, Foundations of differential geometry Vol. I. Reprint of the 1963 original. Wiley Classics Library. A Wiley-Interscience Publication. John Wiley \& Sons, Inc., New York, 1996.

[16] G. D. Mostow, Fully reducible subgroups of algebraic groups, Amer. J. Math. 78 (1956), 200-221.

[17] M. Schneider, On the number of equations needed to describe a variety, Proceedings of Symposia in Pure Mathematics, 41 (1984), 163-180.

[18] A. Toth, D. Varolin, Holomorphic diffeomorphisms of complex semisimple Lie groups, Invent. Math. 139 (2000), no. 2, 351-369.

[19] A. Toth, D. Varolin, Holomorphic diffeomorphisms of semisimple homogenous spaces, Compos. Math. 142 (2006), no. 5, 1308-1326.

[20] D. Varolin, The density property for complex manifolds and geometric structures, J. Geom. Anal. 11 (2001), no. 1, 135-160.

[21] D. Varolin, The density property for complex manifolds and geometric structures II, Internat. J. Math. 11 (2000), no. 6, 837-847.

[22] D. Varolin, A general notion of shears, and applications Michigan Math. J. 46 (1999), no. 3, $533-553$. 
Department of Mathematics, University of Miami, Coral Gables, FL 33124 USA E-mail address: kaliman@math.miami.edu

Mathematisches Institut, Universität Bern, Sidlerstr. 5, CH-3012 Bern, SwitzerLAND

E-mail address: Frank.Kutzschebauch@math.unibe.ch 